\title{
Confluence by Critical Pair Analysis Revisited*
}

\author{
Nao Hirokawa ${ }^{10000-0002-8499-0501]}$, Julian Nagele ${ }^{2[0000-0002-4727-4637]}$, \\ Vincent van Oostrom ${ }^{3}$, and Michio Oyamaguchi ${ }^{4}$ \\ 1 JAIST, Japan, hirokawa@jaist.ac.jp \\ 2 Queen Mary University of London, UK, j.nagele@qmul.ac.uk \\ 3 University of Innsbruck, Austria, Vincent.van-Oostrom@uibk.ac.at \\ 4 Nagoya University, Japan, oyamaguchi@za.ztv.ne.jp
}

\begin{abstract}
We present two methods for proving confluence of left-linear term rewrite systems. One is hot-decreasingness, combining the parallel/development closedness theorems with rule labelling based on a terminating subsystem. The other is critical-pair-closing system, allowing to boil down the confluence problem to confluence of a special subsystem whose duplicating rules are relatively terminating.
\end{abstract}

Keywords: Term rewriting - Confluence - Decreasing diagrams.

\section{Introduction}

We present two results for proving confluence of first-order left-linear term rewrite systems, which extend and generalise three classical results: Knuth and Bendix' criterion [19] and strong and parallel closedness due to Huet [16]. Our idea is to reduce confluence of a term rewrite system $\mathscr{R}$ to that of a subsystem $C$ comprising rewrite rules needed for closing the critical pairs of $\mathcal{R}$. In Section 3 we introduce the notion of a critical-pair-closing system and present a confluence-preservation result based on relative termination $C_{d} / \mathscr{R}$ of the duplicating part $C_{d}$ of $C$. In Section 4 we introduce hot-decreasingness, requiring that critical pairs can be closed using rules that are either below those in the peak or in a terminating subsystem $C$. For the left-linear systems we consider, our first criterion generalises Huet's strong closedness, and the second both his parallel closedness and Knuth and Bendix' criterion. Empirical results to assess viability of our results are reported in Section 5 .

In the last decade various classical confluence results for term rewrite systems have been factored through the decreasing diagrams method 27/29] for proving confluence of abstract rewrite systems, often leading to generalisations along the way: e.g. Felgenhauer's multistep labelling [12] generalises Okui's simultaneous closedness [26], the layer framework 11] generalises Toyama's modularity [32, critical pair systems [15] generalise both orthogonality [30] and Knuth and Bendix' criterion [19], and Jouannaud and Liu generalise, among others [20], parallel closedness, but in a way we do not know how to generalise to development closedness [28. This paper fits into this line of research.

\footnotetext{
* Supported by JSPS KAKENHI Grant Number 17K00011.
} 
Huet's parallel closedness result relies on the notion of overlap whose geometric intuition is subtle 124, and reasoning becomes intricate for development closedness as covered by Theorem 3 . We factor the classical theory of overlaps and critical pairs through the encompassment lattice in which overlapping redexpatterns is taking their join and the amount of overlap between redex-patterns is computed via their meet, thus allowing to reason algebraically about overlaps.

We assume the reader is familiar with term rewriting 81131] in general and confluence methods [19/1629] in particular.

\section{Preliminaries on decreasingness and encompassment}

We recall the key ingredients of the decreasing diagrams method for proving confluence, see 25131/2920, and revisit the classical notion of critical pair, recasting its traditional account 19:16 1] based on redexes (substitution instances of left-hand sides) into one based on redex-patterns (left-hand sides).

Decreasingness Consider an ARS comprising an $I$-indexed relation $\rightarrow=$ $\bigcup_{\ell \in I} \rightarrow_{\alpha}$ equipped with a well-founded strict order $\succ$. We refer to $\{\kappa \in I \mid \ell \succ \kappa\}$ by $\curlyvee \ell$, and to $\curlyvee \ell \cup \curlyvee \kappa$ by $\curlyvee \ell, \kappa$. For a subset $J$ of $I$ we define $\rightarrow_{J}$ as $\bigcup_{\ell \in J} \rightarrow_{\ell}$.

Definition 1. A diagram for a peak $b \ell_{\ell} \leftarrow a \rightarrow_{\kappa} c$ is decreasing if its closing conversion has shape $b \leftrightarrow_{\curlyvee \ell}^{*} \cdot \rightarrow_{\kappa}^{=} \cdot \leftrightarrow_{\curlyvee \ell, \kappa}^{*} \cdot \bar{\ell}^{\leftarrow} \leftarrow \cdot \leftrightarrow_{\curlyvee \kappa}^{*} c$. An ARS in this setting is called decreasing if every peak can be completed into a decreasing diagram.

Theorem 1 (27/29]). An ARS is confluent if it is decreasing. Conversely, every countable ARS that is confluent, is decreasing for some set of indices I.

For completeness, it even suffices that the set of labels $I$ is a doubleton [10].

Critical peaks revisited We introduce clusters as the structures obtained after the matching of the left-hand side of a rule in a rewrite step, but before its replacement by the right-hand side. We use them as a tool to analyse overlaps and critical peaks. To illustrate our notions we use the following running example.

Example 1. In the TRS $\mathscr{R}$ with $\varrho(x): f(f(x)) \rightarrow g(x)$ the term $t=f(f(f(f(a))))$ allows the step $f(\varrho(f(a))): t \rightarrow f(g(f(a)))$ and multistep $\varrho(\varrho(a)): t \rightarrow g(g(a))$.

Here $f(\varrho(f(a)))$ and $\varrho(\varrho(a))$ are so-called proofterms, terms representing proofs of rewritability in rewriting logic 22]31. The source of a proofterm can be computed by the 2nd-order substitution src of the left-hand side of the rule for the rule symbo $\sqrt{5}^{5}(\varrho(f(a)))^{\text {src }}=f(\varrho(f(a)))^{\llbracket \varrho:=\lambda x \cdot f(f(x)) \rrbracket}=f(f(f(f(a))))$, and, mutatis mutandis, the same for the target via tgt. Proofclusters introduced here, abstract from such proofterms allowing to represent the matching and substitution phases of multisteps as well, by means of let-expressions.

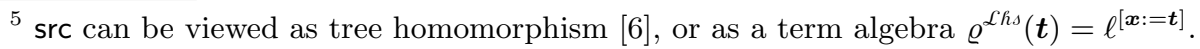


Example 2. The multistep in Example 1 comprises three phases [27, Chapter 4]:

1. let $X, Y=\lambda x . f(f(x)), \lambda y . f(f(y))$ in $X(Y(a))$ denotes matching $f(f(x))$ twice;

2. let $X, Y=\lambda x . \varrho(x), \lambda x . \varrho(x)$ in $X(Y(a))$ denotes replacing by $\varrho$ twice;

3. let $X, Y=\lambda x . g(x), \lambda x . g(x)$ in $X(Y(a))$ denotes substituting $g(x)$ twice.

To represent these we assume to have proofterms $t, s, u, \ldots$ over a signature comprising function symbols $f, g, h, \ldots$, rule symbols $\varrho, \theta, \eta, \ldots, 2$ nd-order variables $X, Y, Z, \ldots$, all having natural number arities, and 1 st-order variables $x, y, z, \ldots$ (with arity 0 ).We call proofterms without 2 nd-order variables or rule symbols, 1 st-order proofterms respectively terms, ranged over by $M, N, L, \ldots$.

Definition 2. A proofcluster is a let-expression let $\boldsymbol{X}=\boldsymbol{Q}$ in $t$, where

- $\boldsymbol{X}$ is a vector $X_{1}, \ldots, X_{n}$ of (pairwise distinct) second-order variables;

- $\boldsymbol{Q}$ is a vector of length $n$ of closed $\lambda$-terms $Q_{i}=\lambda \boldsymbol{x}_{\boldsymbol{i}} . s_{i}$, where $s_{i}$ is a proofterm and the length of the vector $\boldsymbol{x}_{\boldsymbol{i}}$ of variables is the arity of $X_{i}$; and

- $t$ is a proofterm, the body, with its $2 n d$-order variables among $\boldsymbol{X}$.

Its denotation $\llbracket$ let $\boldsymbol{X}=\boldsymbol{Q}$ in $t \rrbracket$ is $t \llbracket \boldsymbol{X}:=\boldsymbol{Q} \rrbracket$. It is a cluster if $\boldsymbol{s}, t$ are terms.

We let $\varsigma, \zeta, \xi, \ldots$ range over (proof)clusters. They denote (proof)terms.

Example 3. Using $\varsigma, \zeta, \xi$ for the three let-expressions in Example 2 , each is a proofcluster and $\varsigma, \xi$ are clusters. Their denotations are the term $\llbracket \varsigma \rrbracket=$ $f(f(f(f(a))))=t$, proofterm $\llbracket \zeta \rrbracket=\varrho(\varrho(a))$, and term $\llbracket \xi \rrbracket=g(g(a))$.

We assume the usual variable renaming conventions, both for the 2nd-order ones in let-binders and the 1st-order ones in $\lambda$-abstractions. We say a proofcluster $\varsigma$ is linear if every (let or $\lambda$ ) binding binds exactly once, and canonical [23] if, when a binding variable occurs to the left of another such (of the same type), then the first bound occurrence of the former occurs before that of the latter in the pre-order walk of the relevant proofterm.

Example 4. Let $\zeta^{\prime}$ and $\xi^{\prime}$ be the clusters let $X=\lambda x . f(f(x))$ in $X(X(a))$ and let $X, Y=\lambda y z . f(f(y)), \lambda x . f(f(x))$ in $Y(X(a, f(a)))$. Each of $\varsigma, \zeta^{\prime}, \xi^{\prime}$ denotes $t$ in Example 1. The cluster $\varsigma$ is linear and canonical, $\zeta^{\prime}$ is canonical but not linear ( $X$ occurs twice in the body), and $\xi^{\prime}$ is neither linear ( $z$ does not occur in $f(y)$ ) nor canonical ( $Y$ occurs outside of $X$ in the body).

We adopt the convention that absent $\lambda$-binders are inserted linearly and canonically; let $X=f(f(x))$ in $X(X(a))$ is $\zeta^{\prime}$. Clusters witness encompassment $\unrhd$.

Proposition 1. $t \unrhd s$ iff $\exists u, X$ s.t. $\llbracket$ let $X=s$ in $u \rrbracket=t$ and $X$ occurs once in $u$.

We define the size $\|t\|$ of a proofterm $t$ in a way that is compatible with encompassment. Formally, $\|t\|$ is the pair comprising the number of non-1st-ordervariable symbols in $t$, and the sum over the 1st-order variables $x$, of the square of the number of occurrences of $x$ in $t$. Then $\|t\|>\|s\|$ if $t \triangleright s$, where we (ab)use $>$ to denote the lexicographic product of the greater-than relation with itself, e.g. $\|g(a, a)\|=(3,0)>\|g(x, x)\|=(1,4)>\|g(x, y)\|=(1,2)$. For a proofcluster 
$\varsigma$ given by let $\boldsymbol{x}=\boldsymbol{s}$ in $t$ its pattern-size $\llbracket \varsigma \|$ is $\sum_{i}\left\|s_{i}\right\|$ (adding component-wise, with empty sum $(0,0))$ and its body-size $\lfloor\varsigma \Downarrow$ is $\|t\|$. Encompassment $\unrhd$ is at the basis of the theory of reducibility [6, Section 3.4.2]: $t$ is reducible by a rule $\ell \rightarrow r$ iff $t \unrhd \ell$. For instance, let $X=f(f(x))$ in $f(X(f(a)))$ is a witness to reducibility of $t$ in Example 1. We call it, or simply $f(f(x))$, a pattern in $t$.

Definition 3. Let $\varsigma$ be a canonical linear proofcluster let $\boldsymbol{X}=s$ in $t$ with term $t$. We say $\varsigma$ is a multipattern if each $s_{i}$ is a non-variable 1 st-order term, and $\varsigma$ is a multistep if each $s_{i}$ has shape $\varrho(\boldsymbol{x})$, i.e. a rule symbol applied to a sequence of pairwise distinct variables. If $\boldsymbol{X}$ has length 1 we drop the prefix 'multi'.

We use $\Phi, \Psi, \Omega, \ldots$ to range over multisteps, and $\phi, \psi, \omega, \ldots$ to range over steps. Taking their denotation yields the usual multistep [3115] and step ARSs $\rightarrow$ and $\rightarrow$ underlying a TRS $R$. These can be alternatively obtained by first applying src and tgt (of which only the former is guaranteed to yield a multipattern, by left-linearity) and then taking denotations: $\llbracket \Phi^{\text {src }} \rrbracket=\llbracket \Phi \rrbracket^{\text {src }}$ and $\llbracket \Phi^{\text {tgt }} \rrbracket=\llbracket \Phi \rrbracket^{\text {tgt }}$. Pattern- and body-sizes of multipatterns are compositional.

Proposition 2. For multipatterns $\varsigma, \varsigma$ if $\varsigma=\varsigma_{0}^{[\boldsymbol{x}:=\varsigma]}$ with each variable among $\boldsymbol{x}$ occurring once in the body of $\varsigma_{0}$, then $\llbracket \varsigma \rrbracket=\sum_{i} \varsigma_{i}$, and $\llbracket \varsigma \Downarrow \geqslant \llbracket \varsigma_{i} \Downarrow$ for all $i$, with strict inequality holding in case the substitution is not a bijective renaming. Here multipattern-substitution substitutes in the body and combines let-bindings.

Multipatterns are ordered by refinement $\sqsubseteq$.

Definition 4. Let $\varsigma$ and $\zeta$ be multipatterns let $\boldsymbol{X}=\boldsymbol{s}$ in $t$ and let $\boldsymbol{Y}=\boldsymbol{u}$ in $w$. We say $\varsigma$ refines $\zeta$ and write $\varsigma \sqsubseteq \zeta$, if there is a 2 nd-order substitution $\sigma$ on $\boldsymbol{Y}$ with $w^{\sigma}=t$ and $\llbracket$ let $\boldsymbol{X}=\boldsymbol{s}$ in $Y_{i}\left(\boldsymbol{y}_{\boldsymbol{i}}\right)^{\sigma} \rrbracket=u_{i}$ for all $i$, with $\boldsymbol{y}_{\boldsymbol{i}}$ the variables of $u_{i}$.

Example 5. We have $\varsigma \sqsubseteq \varsigma^{\prime}$ with $\varsigma^{\prime}$ is let $Z=f(f(f(f(z))))$ in $Z(a)$, and $\varsigma$ as in Example 3 , as witnessed by the 2nd-order substitution mapping $Z$ to $\lambda x . X(Y(x))$.

Lemma 1. $\sqsubseteq$ is a finite distributive lattice [7] on multipatterns denoting a 1storder term $t$, with least element $\perp$ the empty let-expression let $=$ in $t$, and greatest element $\top$ of shape let $X=t^{\prime}$ in $X(\boldsymbol{x})$ with $\boldsymbol{x}$ the vector of variables in $t$.

Proof (Idea). Although showing that $\sqsubseteq$ is reflexive and transitive is easy, showing anti-symmetry or existence of/constructions for meets $\square$ and joins $\sqcup$, directly is not. Instead, it is easy to see that each multipattern let $\boldsymbol{X}=s$ in $t$ is determined by the set of the (non-empty, convex ${ }^{6}$ pairwise disjoint) sets of node positions of its patterns $s_{i}$ in $t$, and vice versa. For instance, the multipatterns $\varsigma$ and $\varsigma^{\prime}$ in Example 5 are determined by $\{\{\varepsilon, 1\},\{1 \cdot 1,1 \cdot 1 \cdot 1\}\}$ and $\{\{\varepsilon, 1,1 \cdot 1,1 \cdot 1 \cdot 1\}\}$. Viewing multipatterns as sets in that way $\varsigma \sqsubseteq \zeta$ iff $\forall P \in \varsigma, \exists Q \in \zeta$ with $P \subseteq Q$. Saying $P, Q \in \varsigma \cup \zeta$ have overlap if $P \cap Q \neq \emptyset$, denoted by $P \curlyvee Q$, characterising meets and joins now also is easy: $\varsigma \sqcap \zeta=\{P \cap Q \mid P \in \varsigma, Q \in \zeta$, and $P \gamma Q\}$, and $\varsigma \sqcup \zeta=\left\{\bigcup P_{\varnothing} \mid P \in \varsigma \cup \zeta\right\}$, where $P_{\varnothing}=\left\{Q \in \varsigma \cup \zeta \mid P \ell^{*} Q\right\}$, i.e. the sets connected

\footnotetext{
${ }^{6}$ Here convex means that for each pair of positions $p, q$ in the set, all positions on the shortest path from $p$ to $q$ in the term tree are also in the set, cf. [31, Definition 8.6.21].
} 
to $P$ by successive overlaps. On this set-representation $\sqsubseteq$ can be shown to be a finite distributive lattice by set-theoretic reasoning, using that the intersection of two overlapping patterns is a pattern again 7 . For instance, $\perp$ is the empty set and $T$ is the singleton containing the set of all non-variable positions in $t$.

The (proof of the) lemma allows to freely switch between viewing multisteps and multipatterns as let-expressions and as sets of sets of positions, and to reason about (non-)overlap of multipatterns and multisteps in lattice-theoretic terms. We show any multistep $\Phi$ can be decomposed horizontally as $\phi$ followed by $\Phi / \phi$ for any step $\phi \in \Phi[1528$, and vertically as some vector $\boldsymbol{\Phi}$ substituted in a prefix $\Phi_{0}$ of $\Phi$, and that peaks can be decomposed correspondingly.

Definition 5. For a pair of multipatterns $\varsigma, \zeta$ denoting the same term its amount of overla $\rrbracket^{8}$ and non-overlap is $\varsigma \cap \zeta=\llbracket \varsigma \sqcap \zeta \prod$ respectively $\varsigma \uplus \zeta=\lfloor\varsigma \sqcup \zeta \Downarrow$, we say $\varsigma, \zeta$ is overlapping if $\varsigma \sqcap \zeta \neq \perp$, and critically overlapping if moreover $\varsigma \sqcup \zeta=\top$ and $\llbracket \varsigma \rrbracket=\llbracket \zeta \rrbracket$ is linear. This extends to peaks $s_{\Phi} \leftarrow-t \rightarrow \rightarrow_{\Psi} u$ via $\Phi^{\mathrm{src}}$ and $\Psi^{\mathrm{src}}$.

Note $\varsigma, \zeta$ is overlapping iff $\varsigma \cap \zeta \neq(0,0)$. Critical peaks $s_{\phi} \leftarrow t \rightarrow_{\psi} u$ are classified by comparing the root-positions $p_{\phi}, p_{\psi}$ of their patterns with respect to the prefix order $\prec_{o}$, into being outer-inner $\left(p_{\phi} \prec_{o} p_{\psi}\right)$, inner-outer $\left(p_{\psi} \prec_{o} p_{\phi}\right)$, or overlay $\left(p_{\psi}=p_{\phi}\right)$, and induce the usual [1916812531] notion of critical pair $\left.(s, u)\right]^{9}$

Definition 6. A pair $\left(\varsigma^{\prime}, \zeta^{\prime}\right)$ of overlapping patterns such that $\varsigma^{\prime}, \zeta^{\prime}$ are in the multipatterns $\varsigma, \zeta$ with $\top=\varsigma \sqcup \zeta$, is called inner, if it is minimal among all such pairs, comparing them in the lexicographic product of $\prec_{o}$ with itself, via the root-positions of their patterns, ordering these themselves first by $\preceq_{o}$. This extends to pairs of steps in peaks of multisteps via src.

Proposition 3. If $(\phi, \psi)$ is an inner pair for a critical peak $\Phi \leftarrow \circ \cdot \multimap \rightarrow$, and $\phi \in \Phi, \psi \in \Psi$ contract redexes at the same position, then $\phi=\Phi$ and $\psi=\Psi$.

For patterns and peaks of ordinary steps, their join being top, entails they are overlapping, and the patterns in a join are joins of their constituent patterns.

Proposition 4. Linear patterns $\varsigma, \zeta$ are critically overlapping iff $\varsigma \sqcup \zeta=\top$.

Lemma 2. If $\xi=\varsigma \sqcup \zeta$ and $\varsigma, \zeta \sqsubseteq \xi$ are witnessed by the 2 nd-order substitutions $\sigma, \tau$, for multipatterns $\varsigma$ and $\zeta$ given by let $\boldsymbol{X}=\boldsymbol{t}$ in $M$ and let $\boldsymbol{Y}=\boldsymbol{s}$ in $N$, then for all let-bindings $Z=u$ of $\xi, \top_{u}=\left(\right.$ let $\boldsymbol{X}=\boldsymbol{t}$ in $\left.Z(\boldsymbol{z})^{\sigma}\right) \sqcup\left(\right.$ let $\boldsymbol{Y}=\boldsymbol{s}$ in $\left.Z(\boldsymbol{z})^{\tau}\right)$.

Lemma 3 (Vertical). A peak $s_{\Phi} \leftarrow t-\iota_{\Psi} u$ of overlapping multisteps either is critical or it can be vertically decomposed as:

$$
s_{0}^{[\boldsymbol{x}:=\boldsymbol{s}]} \Phi_{0}^{[\boldsymbol{x}:=\boldsymbol{\Phi}]} \leftarrow \odot t_{0}^{[\boldsymbol{x}:=\boldsymbol{t}]} \multimap \overbrace{\Psi_{0}^{[\boldsymbol{x}:=\Psi]}} u_{0}^{[\boldsymbol{x}:=\boldsymbol{u}]}
$$

for peaks $s_{i} \Phi_{i} \leftarrow t_{i} \multimap \Psi_{i} u_{i}$ with $\Phi \cap \Psi \geqslant \Phi_{i} \cap \Psi_{i}$ and $\Phi \uplus \Psi>\Phi_{i} \uplus \Psi_{i}$, for all $i$.

\footnotetext{
7 This fails for, e.g., connected graphs; these may fall apart into non-connected ones.

${ }^{8}$ For the amount of overlap for redexes in parallel reduction $\Pi$, see e.g. [16124].

${ }^{9}$ We exclude neither overlays of a rule with itself nor pairs obtained by symmetry.
} 
Let $\Phi, \Psi$ in $s$ s for rules $\varrho_{i}\left(\boldsymbol{x}_{\boldsymbol{i}}\right): \ell_{i} \rightarrow r_{i}$ and $\theta_{j}\left(\boldsymbol{y}_{j}\right): g_{j} \rightarrow d_{j}$. Lemma 1 entails that if $\Phi, \Psi$ are non-overlapping their patterns are (pairwise) disjoint, so that the join $\Phi^{\text {src }} \sqcup \Psi^{\text {src }}$ is given by taking the (disjoint) union of the let-bindings: let $\boldsymbol{X} \boldsymbol{Y}=\boldsymbol{\ell} \boldsymbol{g}$ in $L$ for some $L$ such that $L^{\llbracket \boldsymbol{Y}:=\boldsymbol{g} \rrbracket}=M$ and $L^{\llbracket \boldsymbol{X}:=\ell \rrbracket}=N$. We define the join ${ }^{10} \Phi \sqcup \Psi$ and residual $\Phi / \Psi$ by let $\boldsymbol{X} \boldsymbol{Y}=\boldsymbol{\varrho}(\boldsymbol{x}) \boldsymbol{\theta}(\boldsymbol{y})$ in $L$ respectively let $\boldsymbol{X}=\boldsymbol{\varrho}(\boldsymbol{x})$ in $L^{\llbracket \boldsymbol{Y}:=\boldsymbol{d} \rrbracket}$, where, as substituting the right-hand sides $\boldsymbol{d}$ may lose being linear and canonical, we implicitly canonise and linearise the latter by reordering and replicating let-bindings. As one easily checks, then $t \rightarrow \rightarrow_{\Phi \sqcup \Psi} \cdot \Phi / \Psi \leftarrow u$, giving rise to the classical residual theory [5/17/2]31. We let $\phi \in \Phi$ abbreviate $\exists \Psi . \Phi=\phi \sqcup \Psi$.

Example 6 . The steps $\phi$ and $\psi$ given by let $X=\lambda x . \varrho(x)$ in $X(f(f(a)))$ respectively let $X=\lambda x . \varrho(x)$ in $f(f(Y(a)))$, are non-overlapping, $\phi, \psi \in \zeta, \phi \sqcup \psi=\zeta$, and $f(f(g(a))) \rightarrow \rightarrow_{\phi / \psi} g(g(a))$, for $\zeta$ and $\varrho$ as in Example 3 .

Lemma 4 (Horizontal). A peak $t_{\Phi} \leftarrow \circ \cdot-\rightarrow_{\Psi} s$ of multisteps either

1. is non-overlapping and then $t \rightarrow \rightarrow_{\Psi / \Phi} \cdot \Phi / \Psi \leftarrow 0-s$, with the rule symbols occurring in $\Psi / \Phi$ contained in $\Psi$ (and those in $\Phi / \Psi$ contained in $\Phi$ ); or

2. it can be horizontally decomposed: $t_{\Phi / \phi} \leftarrow \cdot{ }_{\phi} \leftarrow \cdot \rightarrow_{\psi} \cdot \rightarrow \rightarrow_{\Psi / \psi}$ s for some peak ${ }_{\phi} \leftarrow \cdot \rightarrow_{\psi}$ of overlapping steps $\phi \in \Phi$ and $\psi \in \Psi$.

The above allows to refactor the proof of the critical pair lemma for left-linear TRSs, such that the critical peaks form the base case of vertical decomposition, and the induction step uses that joins are closed under composition.

Example 7 ([16]). To show: a left-linear TRS is locally confluent if all critical pairs are joinable. We proceed by induction on the non-overlap-size $\phi \uplus \psi$ of a peak $\bar{\phi}^{\leftarrow} \leftarrow \rightarrow \overline{\bar{\psi}}$ of empty or single steps, ordered by $>$. If the steps do not have overlap, then we conclude by Lemma 4 (1). Otherwise, the peak either is critical in which case we conclude by assumption, or it is not critical, so can be decomposed as in the second item of Lemma 3 into smaller such peaks $\underset{\phi_{i}}{\bar{E}} \leftarrow \cdot \rightarrow \overline{\bar{\psi}}_{i}$. Since these are $>$-smaller, the induction hypothesis yields them joinable, from which we conclude by reductions and joins being closed under composition.

\section{Confluence by critical-pair closing systems}

We introduce a confluence criterion based on identifying for a term rewrite system $\mathscr{R}$ a subsystem $\mathcal{C}$ such that every $\mathcal{R}$-critical peak can be closed by means of $C$-conversions, rendering the rules used in the peak redundant.

Definition 7. A TRS C is critical-pair closing for a TRS R, if $C$ is a subsystem of $\mathscr{R}$ and $s \leftrightarrow^{*} t$ holds for all critical pairs $(s, t)$ of $\mathcal{R}$.

We phrase the main result of this section as a preservation-of-confluence result. We write $\rightarrow_{\mathcal{S} / \mathscr{R}}$ for $\rightarrow_{\mathfrak{R}} \cdot \rightarrow_{\mathcal{S}} \cdot \rightarrow_{\mathscr{R}}$, and if it is terminating, $\delta / \mathscr{R}$ is said to be (relatively) terminating. By $C_{d}$ we denote the set of all duplicating rules in $C$.

${ }^{10}$ This does not create ambiguity with joins of multipatterns since if $\Phi \neq \Psi$, then $\llbracket \Phi \rrbracket \neq \llbracket \Psi \rrbracket$ unless the let-bindings of both are empty, so both are bottom. 
Theorem 2. If $C$ is a critical-pair-closing system for a left-linear TRS $R$ such that $C_{d} / \mathscr{R}$ is terminating, then $\mathcal{R}$ is confluent if $C$ is confluent.

Any left-linear TRS is critical-pair-closing for itself. However, the power of the method relies on choosing small $C$. Before proving Theorem 2, we illustrate it by some (non-)examples and give a special case.

Example 8. Consider the left-linear TRS $\mathscr{R}$ :

$$
\begin{array}{llll}
\varrho_{1}: \text { nats } \rightarrow 0: \operatorname{inc}(\text { nats }) & \varrho_{3}: \quad \operatorname{inc}(x: y) \rightarrow s(x): \operatorname{inc}(y) & \varrho_{5}: h d(x: y) \rightarrow x \\
\varrho_{2}: d(x) \rightarrow x:(x: d(x)) & \varrho_{4}: \operatorname{inc}(t l(\text { nats })) \rightarrow t l(\operatorname{inc}(\text { nats })) & \varrho_{6}: \quad t l(x: y) \rightarrow y
\end{array}
$$

Its subsystem $C=\left\{\varrho_{1}, \varrho_{3}, \varrho_{6}\right\}$ is a critical-pair-closing system for $\mathscr{R}$, as the only $R$-critical pair induced by $\varrho_{4}$ and $\varrho_{1}$ can be $C$-closed:

$$
\begin{aligned}
& t l(\text { inc }(\text { nats })) \longleftarrow \varrho_{4} \quad \operatorname{inc}(t l(\text { nats })) \longrightarrow \varrho_{1} \operatorname{inc}(t l(0: \operatorname{inc}(\text { nats }))) \\
& \varrho_{1} \downarrow \quad \downarrow \varrho_{6} \\
& t l(\operatorname{inc}(0: \operatorname{inc}(\text { nats }))) \underset{\varrho_{3}}{\longrightarrow} t l\left(s(0): \operatorname{inc}(\text { inc }(\text { nats })) \underset{\varrho_{6}}{\longrightarrow} \operatorname{inc}(\text { inc }(\text { nats }))\right.
\end{aligned}
$$

Note that all $C$-rules are linear so $C_{d} / \mathscr{R}$ is vacuously terminating. Thus, by Theorem 2 it is sufficient to show confluence of $C$. Because $C$ has no critical pairs, the empty TRS $\emptyset$ is a critical-pair-closing TRS for $C$. As $\emptyset / C$ is terminating, confluence of $C$ follows from that of $\emptyset$, which is trivial.

Observe how confluence was shown by successive applications of the theorem.

Example 9. Consider the left-linear TRS $R$ :

$$
\begin{array}{rlrl}
\varrho_{1}: f(a, a) & \rightarrow b & \varrho_{3}: f(c, x) \rightarrow f(x, x) & \varrho_{5}: f(c, c) \rightarrow f(a, c) \\
\varrho_{2}: \quad a \rightarrow c & \varrho_{4}: f(x, c) \rightarrow f(x, x) &
\end{array}
$$

Although confluent, $\mathscr{R}$ does not have any confluent critical-pair-closing subsystem $C$ such that $C_{d} / \mathscr{R}$ is terminating, not even $\mathscr{R}$ itself: Because of $b$ being in normal form in the critical pair induced by $b_{\varrho_{1}} \leftarrow f(a, a) \rightarrow_{\varrho_{2}} f(a, c)$, any such subsystem must contain $\varrho_{4}$, as one easily verifies, but $\varrho_{4}$ is both duplicating and non-terminating (looping).

That the condition that $C_{d} / \mathscr{R}$ be terminating cannot be omitted from Theorem 2 , can be seen by considering $\mathscr{R}^{\prime}$ obtained by omitting $\varrho_{5}$ from $\mathscr{R}$. Although $\left\{\varrho_{1}, \varrho_{3}, \varrho_{4}\right\}$ is a confluent critical-pair-closing system for $\mathscr{R}^{\prime}$, it is not confluent.

Remark 1. The example is taken from [15] where it was used to show that decreasingness of critical peaks need not imply that of all peaks, for rule labelling. That example, in turn was adapted from Lévy's TRS in [16] showing that strong confluence need not imply confluence for left-linear TRSs.

The special case we consider is that of TRSs that are ARSs, i.e. where all function symbols are nullary. The identification is justified by that any ARS in the standard sense [25131] can be presented as $\rightarrow_{\mathscr{R}}$ for the TRS $\mathcal{R}$ having a nullary symbol for each object, and a rule for each step of the ARS. Since ARSs have no duplicating rules, Theorem 2 specialises to the following result. 
Corollary 1. If $C$ is critical-pair-closing for $A R S R, R$ is confluent if $C$ is.

Example 10. Consider the TRS $\mathscr{R}$ given by $c \rightarrow a^{\prime} \rightarrow a \rightarrow b$ and $a \rightarrow a^{\prime} \rightarrow c$. It is an ARS having the critical-pair-closing system $C$ given by the first part $c \rightarrow a^{\prime} \rightarrow a \rightarrow b$. Since $C$ is orthogonal it is confluent by Corollary 1, so $\mathcal{R}$ is confluent by the same corollary. In general, a confluent ARS may have many non-confluent critical-pair-closing systems. Requiring local confluence is no impediment to that: The subsystem $C^{\prime}$ of $\mathscr{R}$ obtained by removing $c \rightarrow a^{\prime}$ allows to join all $R$-critical peaks, but is not confluent; it simply is Kleene's example [31, Figure 1.2] showing that local confluence need not imply confluence.

Observe that the ARSs $\mathcal{R}$ and $C^{\prime}$ are isomorphic to the underlying ARSs $\rightarrow \mathcal{R}$ respectively $\rightarrow_{\mathscr{R}^{\prime}}$ of the TRSs in Example 9, when abstracting from the order of the arguments of $f$ and restricting to the component connected of $b$.

For $C_{d} / \mathscr{R}$ to be vacuously terminating it is sufficient that all rules are linear.

Example 11. Consider the linear TRS $\mathcal{R}$ consisting of $\rho_{1}: f(x) \rightarrow f(f(x))$, $\rho_{2}: f(x) \rightarrow g(x)$, and $\rho_{3}: g(x) \rightarrow f(x)$. The subsystem $C=\left\{\rho_{1}, \rho_{3}\right\}$ is criticalpair-closing and has no critical pairs, so $\mathscr{R}$ is confluent.

From the above it is apparent that, whereas usual redundancy-criteria are based on rules being redundant, the theorem gives a sufficient criterion for peaks of steps being redundant.This allows one to leverage the power of extant confluence methods. Here we give but one example, but see our experimental data for many more examples.

Definition 8. A TRS $R$ is strongly closed [16] if $s \rightarrow_{\mathscr{R}} \cdot \overline{\bar{R}} \leftarrow t$ and $s \rightarrow \overline{\bar{R}} \cdot \mathscr{R}^{\sharp}$ $t$ holds for all critical pairs $(s, t)$.

Example 12. Consider the linear TRS $R$ :

$$
\begin{array}{lll}
\varrho_{1}: h(f(x, y)) \rightarrow f(h(r(x)), y) & \varrho_{2}: f(x, k(y, z)) \rightarrow g(p(y), q(z, x)) \\
\varrho_{3}: h(q(x, y)) \rightarrow q(x, h(r(y))) & \varrho_{4}: q(x, h(r(y))) \rightarrow h(q(x, y)) \\
\varrho_{5}: h(g(x, y)) \rightarrow g(x, h(y)) & & \\
\varrho_{6}: a(x, y, z) \rightarrow h(f(x, k(y, z))) & \varrho_{7}: & a(x, y, z) \rightarrow g(p(y), q(z, h(r(x))))
\end{array}
$$

$C=\left\{\varrho_{1}, \ldots, \varrho_{5}\right\}$ is critical-pair-closing for $\mathscr{R}$, since the $\mathscr{R}$-critical peak between $\varrho_{6}$ and $\varrho_{7}$ can be $\varrho$-closed: $h(f(x, k(y, z))) \rightarrow_{\varrho_{1}} f(h(r(x)), k(y, z)) \rightarrow_{\varrho_{2}}$ $g(p(y), q(z, h(r(x))))$. Because $C$ is strongly closed and therefore confluent, see [16, p. 814] for both the example and the confluence result, we obtain $\mathscr{R}$ is confluent.

Remark 2. Neither of the TRSs in Examples 11 and 12 is strongly closed. The former not, because $f(f(x)) \rightarrow_{\mathscr{R}} \cdot \overline{\bar{R}} \leftarrow g(x)$ does not hold, and the latter not because $g(p(y), q(z, h(r(x)))) \rightarrow_{\mathscr{R}} \cdot \overline{\bar{R}} \leftarrow h(f(x, k(y, z)))$ does not hold.

Having illustrated the usefulness of Theorem 2 , we now present its proof. We even give two proofs, although both capture the same intuition. The first is a high-level proof (for the countable case) using the decreasing diagrams technique, whereas the second factors through a concrete lemma for indexed abstract rewrite systems. We first provide the intuition underlying both proofs. 
In TRSs there are two types of peaks: overlapping and non-overlapping ones. As Example 9 shows, confluence criteria only addressing the former need not generalise from ARSs to TRSs. Note that one of the peaks showing non-confluence of $\mathscr{R}^{\prime}$, the one between $\varrho_{2}$ and $\varrho_{3}\left(\varrho_{4}\right)$, is non-overlapping. Therefore, restricting to a subsystem without $\varrho_{2}$ can only provide a partial analysis of confluence of $\mathscr{R}^{\prime}$; the (non-overlapping) interaction between $C$ and $R-C$ is not accounted for, and indeed that is fatal here. The intuition for our proof is that the problem is that the number of such interactions is unbounded due to the presence of the duplicating and non-terminating rule $\varrho_{3}$ (and $\varrho_{4}$ ) in $C$, and that requiring termination of $C_{d} / \mathscr{R}$ bounds that number and suffices to regain confluence.

Proof (of Theorem 2 by decreasing diagrams). Let $C$ be a critical-pair-closing system for $\mathscr{R}$ such that $C_{d} / \mathscr{R}$ is terminating and $\rightarrow_{e}$ is countable. Let $\hat{\mathcal{R}}=\mathscr{R}-C$ and $\hat{C}=C-C_{d}$, so that $\hat{R}, \hat{C}, C_{d}$ forms a partition of (the rules of) $\mathscr{R}$ and $\hat{C}, C_{d}$ of $C$, and consider the following labellings of steps in a conversion:

- a multistep $t \rightarrow \rightarrow_{\hat{R}} s$ is labeled by a triple $(n, \hat{t}, m)$ with $n$ denoting the number of $\hat{R}_{\hat{R}} \leftarrow$-steps to its left in the conversion (symmetrically, $\rightarrow \rightarrow_{\hat{\mathcal{R}}}$-steps to its right for $\hat{\hat{R}} \leftarrow 0$-steps) ${ }^{11} \hat{t}$ a term $\hat{t} \rightarrow \mathfrak{R} t$ (a so-called predecessor [29, Example 18]), and $m$ the maximal length of the development of the multistep;

- $C$-steps are labelled by any decreasing labelling, which exists by completeness of decreasing diagrams (Theorem 1) for countable systems.

By $\succ$ we denote the well-founded order that orders triples for $\hat{\mathcal{R}}$-steps by the lexicographic product of the greater-than relation $>, \rightarrow_{\mathcal{C} / \mathbb{R}}^{+}$, and of $>$again, the labels for $C$-step according to the decreasing labeling, and the former above the latter. We show each local $\mathscr{R}$-peak can be completed into a decreasing diagram, distinguishing cases on steps and on whether their redexes have overlap.

- A peak of shape $\hat{\hat{R}} \leftarrow \bullet \cdot-\rightarrow_{\hat{R}}$ such that the steps do not overlap can, by Lemma 41, be completed by a valley of shape $\rightarrow \rightarrow_{\hat{R}} \cdot \hat{R} \leftarrow 0$. We conclude by a decrement in the first component for both multisteps in the valley.

- By Lemma 42 an overlapping peak of shape $\hat{\mathcal{R}}^{\leftarrow \cdot \cdot-0 \rightarrow_{\hat{\mathcal{R}}}}$ can be horizontally decomposed as $\hat{\mathscr{R}} \leftarrow \cdot \hat{\mathscr{R}} \leftarrow \cdot \rightarrow_{\hat{\mathcal{R}}} \cdot \rightarrow \rightarrow_{\hat{\mathscr{R}}}$ with $\hat{\hat{R}} \leftarrow \cdot \rightarrow_{\hat{\mathscr{R}}}$ an overlapping peak. By $C$ being critical-pair-closing for $\mathscr{R}$, that peak can be closed by a $C$-conversion, so the original peak can be transformed into a conversion of shape $\hat{R} \leftarrow 0$. $\leftrightarrow_{C}^{*} \cdot \multimap \rightarrow_{\hat{R}}$, which is seen to be decreasing: the first and second components of both $\hat{\mathscr{R}}$-multisteps do not change, the first obviously so and the second by choosing to keep the same predecessors, but their third components decrease (by having developed one redex each), and $\mathcal{C}$-steps are smaller than $\hat{\mathcal{R}}$ multisteps;

- A peak of shape $\hat{R} \leftarrow \bullet \cdot \rightarrow c$ such that the steps do not overlap can, by Lemma 4, 1), be completed by a valley of shape $\rightarrow e \cdot \hat{R} \leftarrow \circ$, which is decreasing for the $\mathcal{C}$-steps as these are by definition ordered below $\hat{R}$-multisteps. To see

\footnotetext{
${ }^{11}$ Our peak-transformations preserve these numbers for other steps [20, multi-labels].
} 
decreasingness for the $\hat{R}$-multistep, first observe that the first component does not change. Next, we distinguish cases on the type of the $\mathcal{C}$-step.

If it is a $C_{d}$-step, i.e. it is duplicating, then by choosing its source as second component it decreases.

If it is a $\hat{\mathcal{C}}$-step, i.e. it is linear, they by choosing to keep the same term as second component, all three components are the same, resulting in a decreasing diagram again;

- A peak of shape $\hat{\mathcal{R}} \leftarrow \cdot \rightarrow_{e}$ such that the steps are overlapping can, by the special case of Lemma 4 where one of the multisteps is a single step, be horizontally decomposed as $\hat{\mathcal{R}} \leftarrow \cdot \cdot \hat{\hat{R}} \leftarrow \cdot \rightarrow_{e}$ with $\hat{\hat{R}}_{\hat{R}} \leftarrow \cdot \rightarrow_{e}$ an overlapping peak. Since $C$ is by assumption a subsystem of $\mathscr{R}$, that peak is an $\mathscr{R}$-peak and we may proceed as in the second item.

Instead of relying on completeness of decreasing diagrams, Theorem 1 and thereby on $C$ being countable for defining the order $\succ$, we can instead make $C$-confluence explicit in the form of the diamond property of $\rightarrow e$ :

Lemma 5. Let $\rightarrow_{\mathcal{A}}=\bigcup_{a \in I} \rightarrow_{a}$ be a relation equipped with a well-founded order $\succ$ on a label set $I$, and let $\rightarrow_{\mathcal{B}}$ be a confluent relation with $\rightarrow_{\mathbb{B}} \subseteq \rightarrow_{\mathcal{A}}$. The relation $\rightarrow_{\mathcal{A}}$ is confluent if

1. $a \leftarrow \cdot \rightarrow_{b} \subseteq\left(\rightarrow_{\mathcal{A}} \cdot \mathcal{A} \leftarrow\right) \cup \bigcup_{\{a, b\} \succ_{\text {mul }}\left\{a^{\prime}, b^{\prime}\right\}}\left(a^{\prime} \leftarrow \cdot \leftrightarrow_{\mathcal{B}}^{*} \cdot \rightarrow_{b^{\prime}}\right)$ for all $a, b \in I$; and

2. $a \leftarrow \cdot \rightarrow_{\mathcal{B}} \subseteq\left(\rightarrow_{\mathcal{B}} \cdot a \leftarrow\right) \cup \bigcup_{a \succ a^{\prime}}\left(\rightarrow_{\mathcal{B}} \cdot a^{\prime} \leftarrow \cdot \leftrightarrow_{\mathcal{B}}^{*}\right)$ for all $a \in I$.

Here $\succ_{\text {mul }}$ stands for the multiset extension of $\succ$.

Proof (Sketch). Let $\longmapsto=\rightarrow_{\mathbb{B}} \cdot \rightarrow_{\mathcal{A}}$. We claim that ${ }_{a} \leftarrow \cdot \rightarrow_{\mathcal{B}}^{m} \cdot{ }_{\mathbb{B}}^{n} \leftarrow \cdot \rightarrow_{b} \subseteq \longmapsto \cdot \longleftrightarrow$ holds for all labels $a, b$ and numbers $m, n \geqslant 0$. The claim is shown by wellfounded induction on $(\{a, b\}, m+n)$ with respect to the lexicographic product of $\succ_{\text {mul }}$ and the greater-than order $>$ on $\mathbb{N}$. Thus, the diamond property of $\succ$ follows from the claim and confluence of $\mathscr{B}$. As $\rightarrow_{\mathcal{A}} \subseteq \longmapsto \subseteq \rightarrow_{\mathcal{A}}$, we conclude confluence of $\mathcal{A}$ by e.g. [31, Proposition 1.1.11].

Proof (of Theorem 2 by Lemma句). Let $I$ comprise pairs of a term and a natural number, and define $t \rightarrow_{(\hat{t}, n)} s$ if $t \rightarrow_{\mathcal{R}} t \rightarrow_{\mathfrak{R}} s$ with $n$ the maximal length of a development of the multistep 12 and $\rightarrow_{\mathcal{B}}=\rightarrow_{\mathcal{C}}$, in Lemma 5 . As well-founded order $\succ$ on indices we take the lexicographic product of $\mathcal{C}_{d} / \mathscr{R}$ and greaterthan $>$. Henceforth the proof and its case analysis of peaks follows the above decreasing-diagrams-based proof. Because of this, we only present the interesting case, leaving the others to the reader:

- Suppose $s_{(\hat{t}, n)} \leftarrow t \rightarrow_{C} u$ where the steps do not have overlap. Then by Lemma 4(1), $s \rightarrow_{C} \cdot \mathfrak{R} \leftarrow u$, so $s \rightarrow_{C} \cdot \mathfrak{R} \leftarrow a-u$. Distinguish cases on the type of the $C$-rule employed in $t \rightarrow_{e} u$.

If the rule is duplicating, then $s \rightarrow_{C} \cdot(u, m) \leftarrow u$ for $m$ the maximal length of a development of the $\rightarrow \rightarrow R^{-s t e p}$ from $u$, and condition 2 is satisfied as $t \rightarrow e_{d} u$ implies $(\hat{t}, n) \succeq(u, m)$.

${ }^{12}$ By the Finite Developments Theorem lengths of such developments are finite [31]. 
If the rule is non-duplicating, then $s \rightarrow_{C^{\cdot}}{ }_{(\hat{t}, n)} \leftarrow u$ as $\hat{t} \rightarrow_{\mathscr{R}} t \rightarrow_{\mathscr{R}} u$ by assumption and the length of the maximal development of the residual multistep does not increase when projecting over a linear rule. Again, condition 2 is satisfied.

\section{Confluence by hot-decreasingness}

Linear TRSs have a critical-pair criterion for so-called rule-labelling [29|15|35]: If all critical peaks are decreasing with respect some rule-labelling, then the TRS is decreasing, hence confluent. We introduce the hot-labelling extending that result to left-linear TRSs. To deal with non-right-linear rules we make use of a rule-labelling for multisteps that is invariant under duplication, cf. [12 35].

Remark 3. Naïve extensions fail. Non-left-linear TRSs need not be confluent even without critical pairs [31, Exercise 2.7.20]. That non-right-linear TRSs need not be confluent even if all critical peaks are decreasing for rule-labelling, is witnessed by [15, Example 8] ( $\mathscr{R}^{\prime}$ in Example 9 above).

Definition 9. For a TRS $R$, terminating subsystem $C$, and labelling of $R-C$ -

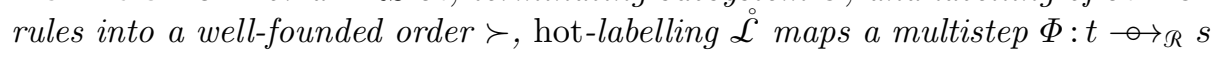

- to the term $t$ if $\Phi$ contains $\mathcal{C}$-rules only; and

- to the set of $\succ$-maximal $R-C$-rules in $\Phi$ otherwise.

The hot-order $\succ$ relates terms by $\rightarrow_{C}^{+}$, sets by $\succ_{\text {mul }}$, and all sets to all terms.

Note $\succ$ is a well-founded order as series composition [3] of $\rightarrow_{C}^{+}$and $\succ_{\text {mul }}$, which are well-founded orders by the assumptions on $C$ and $\succ$. Taking the set of maximal rules in a multistep makes hot-labelling invariant under duplication.

Definition 10. A TRS $R$ is hot-decreasing if its critical peaks are decreasing for the hot-labelling, for some $C$ and $\succ$, such that each outer-inner critical peak $\ell \leftarrow \cdot \rightarrow$ for label $\ell$, is decreasing by a conversion of shape (oi): $\leftrightarrow_{\dot{\vee} \ell}^{*} \cdot$ ir $\ell \leftarrow$.

Theorem 3. A left-linear TRS is confluent, if it is hot-decreasing.

Before proving Theorem 3 , we give (non-)examples and special cases.

Example 13. Taking $C=\emptyset$, labelling rules by themselves, and ordering $\varrho_{4} \succ$ $\varrho_{1}, \varrho_{3}, \varrho_{6}$ in Example 8 , the only critical peak $\left\{\varrho_{4}\right\} \leftarrow \cdot \rightarrow_{\left\{\varrho_{1}\right\}}$ is hot-decreasing as $\rightarrow\left\{\varrho_{1}\right\} \cdot \rightarrow_{\left\{\varrho_{3}\right\}} \cdot \rightarrow_{\left\{\varrho_{6}\right\}} \cdot\left\{\varrho_{6}\right\} \leftarrow$. The peak is outer-inner so the conversion must be of (oi)-shape $\leftrightarrow_{\curlyvee}^{*}\left\{\varrho_{4}\right\} \cdot$ ir $\left\{\varrho_{4}\right\} \leftarrow^{\circ}$. It is, so the system is confluent by Theorem 3 .

Example 14. Since $b$ is an $\mathscr{R}$-normal form in Example 9, the only way to join the outer-inner critical peak $b \varrho_{1} \leftarrow f(a, a) \rightarrow_{\varrho_{2}} f(c, a)$ is by a conversion starting with a step $b \varrho_{1} \leftarrow f(a, a)$. As its label must be identical to the same step in the peak, not smaller, whether we choose $\varrho_{1}$ to be in $C$ or not, the peak is not hot-decreasing, so Theorem 3 does not apply. It shouldn't as restricting to the non-confluent TRS $\mathscr{R}^{\prime}$ in Example 9, the only other critical peak is trivial. 
A special case of Theorem 3, is that a left-linear terminating TRS is confluent [19], if each critical pair is joinable, as can be seen by setting $C=\mathscr{R}$.

Corollary 2. A left-linear development closed TRS is confluent [28, Cor. 24].

Proof. A TRS is development closed if for every critical pair $(t, s)$ such that $t$ is obtained by an outer step, $t \leftarrow a-s$ holds. Taking $C=\emptyset$ and labelling all rules the same, say by 0 , yields that each outer-inner or overlay critical peak is labelled as $t_{\{0\}} \leftarrow \cdot \rightarrow_{\{0\}} s$, and can be completed as $t_{\{0\}} \leftarrow s$, yielding a hot-decreasing diagram of (oi)-shape. We conclude by Theorem 3

The proof of Theorem 3 uses the following structural properties of decreasing diagrams specific to the hot-labelling. The labelling was designed so they hold.

Lemma 6. 1. If the peak $s$ et $t \rightarrow \rightarrow_{\kappa} u$ is hot-decreasing, then it can be completed into a hot-decreasing diagram of shape $s \leftrightarrow_{\curlyvee \ell}^{*} s^{\prime} \rightarrow \rightarrow_{\kappa} s^{\prime \prime} \leftrightarrow_{\curlyvee}^{*} \ell \kappa$ $u^{\prime \prime} \ell \leftarrow 0-u^{\prime} \leftrightarrow_{\curlyvee \kappa}^{*} u$ such that the 1 st-order variables in all terms in the diagram are contained in those of $t$.

2. If the multisteps $\Phi, \Psi$ in the peak $s \Phi \leftarrow 0 t \rightarrow \Psi$ u are non-overlapping, then the valley $s \rightarrow \rightarrow_{\Psi / \Phi} \cdot \Phi / \Psi \leftarrow$ u completes it into a hot-decreasing diagram.

3. If the peak $s \leftarrow 0 \quad t \rightarrow u$ and vector of peaks $\boldsymbol{s} \leftarrow \boldsymbol{t} \boldsymbol{t} \rightarrow \boldsymbol{u}$ have hotdecreasing diagrams, so does the composition $s^{[\boldsymbol{x}:=\boldsymbol{s}]} \leftarrow 0 t^{[\boldsymbol{x}:=\boldsymbol{t}]} \rightarrow u^{[\boldsymbol{x}:=\boldsymbol{u}]}$.

The proof of Theorem 3 is as outlined in Example 7 but refining it into an outer induction based on horizontal decomposition (Lemma 4) decreasing the amount of overlap (ก) between the multisteps, and an inner induction based on vertical decomposition (Lemma 3) decreasing their amount of non-overlap (ש).

Proof (of Theorem 3). We show that every peak $s_{\Phi} \leftarrow t \rightarrow \rightarrow u$ of multisteps $\Phi$ and $\Psi$ can be closed into a hot-decreasing diagram, by induction on the pair $(\Phi \cap \Psi, \Phi \uplus \Psi)$ ordered by the lexicographic product of $>$ with itself. We distinguish cases on whether or not $\Phi$ and $\Psi$ have overlap.

If $\Phi$ and $\Psi$ do not have overlap, then by Lemma 44 1) we have $s \rightarrow \rightarrow_{\Psi / \Phi} \cdot \Phi / \Psi \leftarrow$ $u$. This valley completes the peak into a hot-decreasing diagram by Lemma 6.2.

If $\Phi$ and $\Psi$ do have overlap, then we further distinguish cases on whether or not the overlap is critical.

If the overlap is not critical, then by Lemma 3 the peak can be vertically decomposed into a number of peaks between multisteps $\Phi_{i}, \Psi_{i}$ that have an amount of overlap that is not greater, $\Phi$ ก $\Psi \geqslant \Phi_{i}$ ก $\Psi_{i}$, and a strictly smaller amount of non-overlap $\Phi \uplus \Psi>\Phi_{i} \uplus \Psi_{i}$. Hence the I.H. applies and yields that each such peak can be completed into a hot-decreasing diagram. We conclude by vertically recomposing them yielding a hot-decreasing diagram by Lemma 6 30.

If the overlap is critical, then by Lemma 4 the peak can be horizontally decomposed as $s_{\Phi / \phi} \leftarrow s^{\prime} \phi^{\leftarrow} t \rightarrow_{\psi} u^{\prime} \rightarrow_{\Psi / \psi} u$ for some peak $s^{\prime} \phi^{\leftarrow} t \rightarrow_{\psi} u^{\prime}$ of overlapping steps $\phi \in \Phi$ and $\psi \in \Psi$, i.e. such that $\Phi=\phi \sqcup \Phi^{\prime} \Psi=\psi \sqcup \Psi^{\prime}$ for some $\Phi^{\prime}, \Psi^{\prime}$. We choose $(\phi, \psi)$ to be inner among such overlapping pairs (see Definition 6), assuming w.l.o.g. that $p_{\phi} \preceq_{o} p_{\psi}$ for the root-positions $p_{\phi}, p_{\psi}$ of their patterns. We distinguish cases on whether or not $p_{\phi}$ is a strict prefix of $p_{\psi}$. 


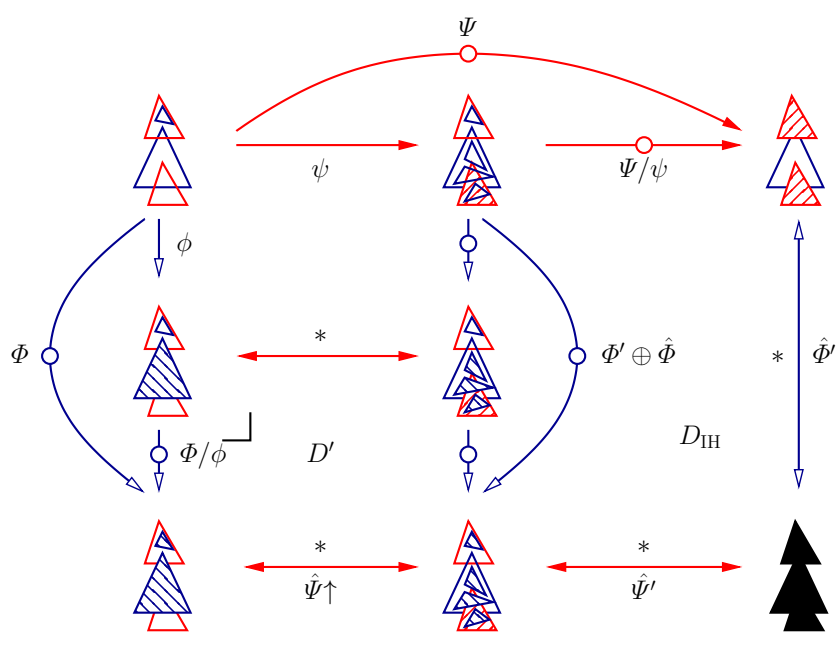

Fig. 1. Outer-inner critical peak construction

If $p_{\phi}=p_{\psi}$, then $\phi=\Phi$ and $\psi=\Psi$ by Proposition 3, so the peak $i s$ overlay, from which we conclude since such peaks are hot-decreasing by assumption.

Suppose $p_{\phi} \prec_{o} p_{\psi}$. We will construct a hot-decreasing diagram $D$ for the peak $s \Phi \leftarrow 0 t-\neg \Psi u$ out of several smaller such diagrams as illustrated in Figure 1. using the multipattern $\varsigma=\Phi^{\text {src }} \sqcup \psi^{\text {src }}$ as a basic building block; it has as patterns those of $\Phi^{\prime}$ and the join of the patterns of $\phi, \psi$. To make $\varsigma$ explicit, unfold $\Phi$ and $\Psi$ to let-expressions let $\boldsymbol{X}=\boldsymbol{\varrho}(\boldsymbol{x})$ in $M$ respectively let $\boldsymbol{Y}=\boldsymbol{\theta}(\boldsymbol{y})$ in $N$, for rules of shapes $\varrho_{i}\left(\boldsymbol{x}_{\boldsymbol{i}}\right): \ell_{i} \rightarrow r_{i}$ and $\theta_{j}\left(\boldsymbol{y}_{j}\right): g_{j} \rightarrow d_{j}$. We let $\boldsymbol{X}=\boldsymbol{X}^{\prime} X$ and $\boldsymbol{Y}=\boldsymbol{Y}^{\prime} Y$ be such that $X$ and $Y$ are the 2nd-order variables corresponding to $\phi \in \Phi$ and $\psi \in \Psi$ for rules $\varrho(\boldsymbol{x}): \ell \rightarrow r$ and $\theta(\boldsymbol{y}): g \rightarrow d$. By the choice of $(\phi, \psi)$ as inner, $\phi^{\text {src }}$ is the unique pattern in $\Phi^{\text {src }}$ overlapping $\psi^{\text {src }}$. As a consequence we can write $\varsigma$ as let $\boldsymbol{X}^{\prime} Z=\boldsymbol{\ell}^{\prime} \hat{t}$ in $L$, for some pattern $\hat{t}$, the join of the patterns of $\phi, \psi$, such that $\sigma$ maps $Z$ to a term of shape $X\left(\boldsymbol{g}_{\boldsymbol{\psi}}\right)$ since $\phi$ is the outer step, and $\tau$ maps it to a term of shape $C\left[Y\left(\boldsymbol{\ell}_{\boldsymbol{\phi}}\right)\right]$, where $\sigma, \tau$ witness $\Phi^{\text {src }}, \psi^{\text {src }} \sqsubseteq \varsigma$. That the other 2 ndorder variables are $\boldsymbol{X}^{\prime}$ follows by $\sigma$ being the identity on them (their patterns do not overlap $\psi$ ), and that these are bound to the patterns $\boldsymbol{\ell}^{\prime}$ by $\tau$ mapping them to 1st-order terms (only $Z$ can be mapped to a non-1st-order term).

We start with constructing a hot-decreasing diagram $\stackrel{\circ}{D}$ for the critical peak $\hat{s}_{\hat{\phi}} \leftarrow \hat{t} \rightarrow_{\hat{\psi}} \hat{u}$ encompassed by the peak between $\phi$ and $\psi$, as follows. We set $\hat{\phi}$ and $\hat{\psi}$ to let $X=\varrho(\boldsymbol{x})$ in $Z(\boldsymbol{z})^{\sigma}$ respectively let $Y=\theta(\boldsymbol{y})$ in $Z(\boldsymbol{z})^{\tau}$. This yields a peak as desired, which is outer-inner as $p_{\hat{\phi}} \prec_{o} p_{\hat{\psi}}$ by $p_{\phi} \prec_{o} p_{\psi}$, and critical by Lemma 2 , hence by the hot-decreasingness assumption, it can be completed into a

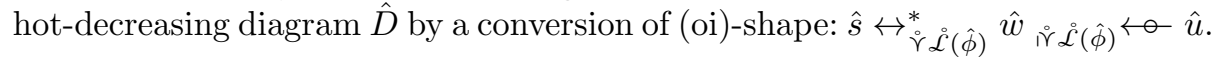
Below we refer to its conversion and multistep as $\hat{\Psi}$ and $\hat{\Phi}$. Based on $\hat{D}$ we construct a hot-decreasing diagram $D^{\prime}$ (Figure 1] left) for the peak $s_{\Phi} \leftarrow 0-t \rightarrow_{\psi}$ 
$u^{\prime}$ by constructing a conversion $\hat{\Psi} \uparrow: s \leftrightarrow^{*} w^{\prime \prime}$ and a multistep $\Phi^{\prime} \oplus \hat{\Phi}: u^{\prime} \multimap w^{\prime \prime}$, with their composition (reversing the latter) of (oi)-shape.

The conversion $\hat{\Psi} \uparrow: s \leftrightarrow^{*} w^{\prime \prime}$ is constructed by lifting the closing conversion $\hat{\Psi}$ of the diagram $\hat{D}$ back into $\varsigma$. Formally, for any multistep $\hat{\Omega}$ given by let $\hat{\boldsymbol{Z}}=\boldsymbol{\eta}(\boldsymbol{w})$ in $\hat{L}$ for rules $\eta_{k}\left(\boldsymbol{w}_{\boldsymbol{k}}\right)$, occurring anywhere in $\hat{\Psi}$, we define its lifting $\hat{\Omega} \uparrow$ to be let $\hat{\boldsymbol{Z}}=\boldsymbol{\eta}(\boldsymbol{w})$ in $L^{\left[\boldsymbol{X}^{\prime}, Z:=\boldsymbol{r}^{\prime}, \hat{L}\right]}$. That is, we update $\varsigma$ by substituting ${ }^{13}$ both $\hat{\Omega}$ (for $Z$, instead of binding that to $\hat{t}$ ) and the right-hand sides $\boldsymbol{r}^{\prime}$ in its body. Because right-hand sides $\boldsymbol{r}$ need not be linear, the resulting proofclusters may have to be linearised (by replicating let-bindings) first to obtain multisteps. This extends to terms $p$ by $p \uparrow=\llbracket($ let $=$ in $p) \uparrow \rrbracket$. That this yields multisteps and terms that connect into a conversion $s=\hat{s} \uparrow \leftrightarrow_{\hat{\Psi} \uparrow}^{*} \hat{w} \uparrow=w^{\prime \prime}$ as desired follows by computation. E.g., $s=M^{\left[\boldsymbol{X}^{\prime}, X:=\boldsymbol{r}^{\prime}, r\right]}=L^{\left[\boldsymbol{X}^{\prime}, Z:=\boldsymbol{r}^{\prime}, \hat{s}\right]}=\hat{s} \uparrow$ using that $\sigma$ witnesses $\Phi^{\text {src }} \sqsubseteq \varsigma$ so that $M=L^{\sigma}$ and $\hat{s}=\llbracket$ let $X=r$ in $Z(\boldsymbol{z})^{\sigma} \rrbracket$. That the labels in $\hat{\Psi} \uparrow$ are strictly below $\mathscr{L}(\Phi)$ follows for set-labels from that lifting clearly does not introduce rule symbols and from that labels of rule symbols in $\hat{\Psi}$ are, by assumption, strictly below the label of the rule $\varrho$ of $\phi$. In case $\Phi$ is term-labelled, by $t$, it follows from closure of $\rightarrow e$-reduction under lifting (which also contracts $\Phi^{\prime}$ ).

The multistep $\Phi^{\prime} \oplus \hat{\Phi}: u^{\prime} \rightarrow w^{\prime \prime}$ is the combination of the multisteps $\Phi^{\prime}$ (the redex-patterns in $\Phi$ other than $\phi)$ and $\hat{\Phi}$, lifting the latter into $\varsigma$. For $\hat{\Phi}: \hat{u} \rightarrow$ $\hat{w}$ given by let $\hat{\boldsymbol{X}}=\hat{\varrho}(\hat{\boldsymbol{x}})$ in $\hat{M}$, it is defined as let $\boldsymbol{X}^{\prime} \hat{\boldsymbol{X}}=\varrho(\boldsymbol{x})^{\prime} \hat{\varrho}(\hat{\boldsymbol{x}})$ in $L^{[Z:=\hat{M}]}$. Per construction it only contracts rules in $\Phi^{\prime}, \hat{\Phi}$, so has a label in $i^{\circ} \dot{\mathcal{L}}(\hat{\Phi})$ by $\Phi=\phi \sqcup \Phi^{\prime}$ and the label of $\hat{\Phi}$ is in $i^{\circ} \dot{L}(\hat{\phi})$ by the (oi)-assumption. That $\Phi^{\prime} \oplus$ $\hat{\Phi}: u^{\prime} \rightarrow w^{\prime \prime}$ follows again by computation, e.g. $\llbracket$ let $\boldsymbol{X}^{\prime} \hat{\boldsymbol{X}}=\boldsymbol{r}^{\prime} \hat{\boldsymbol{r}}$ in $L^{[Z:=\hat{M}]} \rrbracket=$ $L^{\left[\boldsymbol{X}^{\prime}, Z:=\boldsymbol{r}^{\prime}, \hat{M}^{[\hat{\boldsymbol{X}}:=\hat{\boldsymbol{r}}]}\right]}=L^{\left[\boldsymbol{X}^{\prime}, Z:=\boldsymbol{r}^{\prime}, \hat{w}\right]}=\hat{w} \uparrow=w^{\prime \prime}$.

Finally, applying the I.H. to the peak $w_{\Phi^{\prime} \oplus \hat{\Phi}^{\leftarrow}}^{\leftarrow} u^{\prime} \hookrightarrow_{\Psi / \psi} u$ yields some hot-decreasing diagram $D_{\mathrm{IH}}$ (Figure 1, right). Prefixing $\hat{\Psi} \uparrow$ to its closing conversion between $w^{\prime \prime}$ and $u$, then closes the original peak $s$ s into a hot-decreasing diagram $D$, because labels of steps in $\hat{\Psi} \uparrow$ are in $\dot{\Upsilon} \dot{\mathcal{L}}(\Phi)$, $\dot{\mathcal{L}}(\Phi) \succeq \dot{\mathcal{L}}\left(\Phi^{\prime} \oplus \hat{\Phi}\right)$ as seen above, and $\dot{\mathcal{L}}(\Psi) \succeq \dot{\mathcal{L}}(\Psi / \psi)$. The I.H. applies since $\Phi \cap \Psi>\left(\Phi^{\prime} \oplus \hat{\Phi}\right) \cap(\Psi / \psi)$ : To see this, we define $L^{\prime}=L^{\left[\boldsymbol{Z}^{\prime}:=\ell^{\prime}\right]}$ and $F^{\prime}=$ let $\hat{\boldsymbol{X}}=\hat{\boldsymbol{\ell}}$ in $L^{\prime[Z:=\hat{M}]}$ and collect needed ingredients (the joins are disjoint):

$$
\begin{aligned}
& D=\Phi^{\text {src }} \quad=\left(\text { let } \boldsymbol{X}^{\prime}=\boldsymbol{\ell}^{\prime} \text { in } L^{[Z:=\hat{t}]}\right) \sqcup \phi^{\text {src }}=\Phi^{\text {src }} \sqcup \phi^{\text {src }} \\
& E=\Psi^{\text {src }} \quad=\left(\text { let } \boldsymbol{Y}^{\prime}=\boldsymbol{g}^{\prime} \text { in } N^{[Y:=g]}\right) \sqcup \psi^{\text {src }}=\Psi^{\prime \text { src }} \sqcup \psi^{\text {src }} \\
& D^{\prime}=\left(\Phi^{\prime} \oplus \hat{\Phi}\right)^{\text {src }}=\left(\text { let } \boldsymbol{X}^{\prime}=\boldsymbol{\ell}^{\prime} \text { in } L^{[Z:=\hat{u}]}\right) \sqcup F^{\prime} \\
& E^{\prime}=(\Psi / \psi)^{\text {src }}=\text { let } \boldsymbol{Y}^{\prime}=\boldsymbol{g}^{\prime} \text { in } N^{[Y:=d]}
\end{aligned}
$$

Using these one may reason with sets of patterns (not let-expressions as $t \neq s^{\prime}$; the sets are positions in both $\left.t, s^{\prime}\right)$ as follows, relying on distributivity:

$$
(D \sqcap E) \sqsupset\left(D_{-} \sqcap E\right)=\left(D_{-} \sqcap E^{\prime}\right)=\left(D_{+}^{\prime} \sqcap E^{\prime}\right) \sqsupseteq\left(D^{\prime} \sqcap E^{\prime}\right)
$$

where $F$ is the singleton $\left\{\left\{p \in \phi^{\text {src }} \mid p_{\psi} \npreceq p\right\}\right\}$ having all positions in $\phi$ not below $\psi$ 's root, $D_{-}=\Phi^{\text {src }} \sqcup F$, and $D_{+}^{\prime}=\left(D^{\prime}-F^{\prime}\right) \sqcup F$.

${ }^{13}$ For this to be a valid 2nd-order substitution, the 1st-order variables of $\hat{\Omega}(\hat{L})$ must be contained in those of $\hat{t}$, which we may assume by Lemma 61. 
Table 1. Experimental results

\begin{tabular}{lccccc}
\hline & Thm. [2(i) & Thm. 2(ii) & Thm. 3 & dc & sc \\
\# proved (\# timeouts) & $80(19)$ & $95(14)$ & $89(38)$ & $34(1)$ & $62(1)$ \\
\hline
\end{tabular}

\section{Implementation and experiments}

The presented confluence techniques have been implemented in the confluence tool Saigawa version 1.10 [13. We used the tool to test the criteria on 432 leftlinear TRSs in COPS [14] Nos. 1-1036, where we ruled out duplicated problems. Out of 432 systems, 224 are known to be confluent and 173 are non-confluent.

We briefly explain how we automated the presented techniques. As illustrated in Examples 8 and 12 , Theorem 2 can be used as (i) a stand-alone criterion and also as (ii) a generalisation of strong closedness. The condition $s \rightarrow_{\mathscr{R}}^{*} \cdot \overline{\bar{R}} \leftarrow t$ of strong closedness is tested by $s \rightarrow \underset{\mathcal{R}}{\leqslant 5} \cdot \overline{\bar{R}} \leftarrow t$. For a critical peak $s \ell \leftarrow \cdot \rightarrow$ $t$, hot-decreasingness is checked by the disjunction of $s \rightarrow \underset{\sim}{<} \cdot$ ir $\ell \leftarrow 0 t$ and $s \rightarrow e \cdot i r \leftarrow a t$ if it is outer-inner one, and if it is overlay, the disjunction of

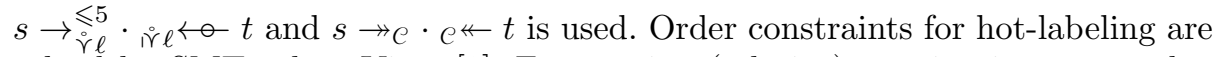
solved by SMT solver Yices [9]. For proving (relative) termination we employ the termination tool NaTT version 1.8 [34]. Finally, suitable subsystems $C$ used in our criteria are searched by enumeration.

Table 1 gives a summary of the results ${ }^{14}$ The tests were run on a PC equipped with Intel Core i7-5500U CPU $(2.4 \mathrm{GHz})$ and $8 \mathrm{~GB}$ memory using a timeout of 60 seconds. For the sake of comparison we also tested the strong closedness theorem (sc) and development closedness theorem (dc). As theoretically expected, they are subsumed by their generalizations.

\section{Conclusion and future work}

We presented two methods for proving confluence of TRSs, dubbed critical-pairclosing systems and hot-decreasingness. We gave a lattice-theoretic characterisation of overlap. Since many results in term rewriting, and beyond, are based on reasoning about overlap, which is notoriously hard [24], we expect that formalising our characterisation could simplify or even enable formalising them. We expect that both methods generalise to commutation, extend to HRSs [21, and can be strengthened by considering rule specialisations.

Example 15. Analysing the TRS $\mathscr{R}$ of Example 9 one observes that for closing the critical pairs only (non-duplicating) instances of the duplicating rules $\varrho_{3}$ and $\varrho_{4}$ are used. Adjoining these specialisations allows the method to proceed: Adjoining $\varrho_{3}(a): f(c, a) \rightarrow f(a, a)$ and $\varrho_{4}(a): f(a, c) \rightarrow f(a, a)$ to $\mathscr{R}$ yields a (reductionequivalent) TRS having critical-pair-closing system $\left\{\varrho_{1}, \varrho_{3}(a), \varrho_{4}(a), \varrho_{5}\right\}$. Since this is a linear system without critical pairs, it is confluent, so $\mathscr{R}$ is as well.

\footnotetext{
$\overline{14}$ Detailed data are available from: http://www.jaist.ac.jp/project/saigawa/19cade/
} 


\section{References}

1. Baader, F., Nipkow, T.: Term Rewriting and All That. Cambridge University Press (1998)

2. Barendregt, H.: The Lambda Calculus: Its Syntax and Semantics, Studies in Logic and the Foundations of Mathematics, vol. 103. North-Holland (1985)

3. Bechet, D., de Groote, P., Retoré, C.: A complete axiomatisation for the inclusion of series-parallel partial orders. In: Comon, H. (ed.) Proc. 8th RTA. LNCS, vol. 1232, pp. 230-240. Springer Berlin Heidelberg, Berlin, Heidelberg (1997)

4. Boudol, G.: Computational semantics of term rewriting systems. In: Nivat, M., Reynolds, J. (eds.) Algebraic Methods in Semantics, pp. 169-236. Cambridge University Press (1985)

5. Church, A., Rosser, J.: Some properties of conversion. Transactions of the American Mathematical Society 39, 472-482 (1936)

6. Comon, H., Dauchet, M., Gilleron, R., Löding, C., Jacquemard, F., Lugiez, D., Tison, S., Tommasi, M.: Tree Automata Techniques and Applications. http://www.grappa.univ-lille3.fr/tata (2007)

7. Davey, B., Priestley, H.: Introduction to Lattices and Order. Cambridge University Press (1990)

8. Dershowitz, N., Jouannaud, J.P.: Rewrite systems. In: van Leeuwen, J. (ed.) Handbook of Theoretical Computer Science, vol. B, Formal Models and Semantics, pp. 243-320. Elsevier (1990)

9. Dutertre, B.: Yices 2.2. In: Proc. 26th CAV. LNCS, vol. 8559, pp. 737-744 (2014)

10. Endrullis, J., Klop, J., Overbeek, R.: Decreasing diagrams with two labels are complete for confluence of countable systems. In: Proc. 3rd FSCD. LIPIcs, vol. 108, pp. 14:1-14:15 (2018), http://dx.doi.org/10.4230/LIPIcs.FSCD.2018.14

11. Felgenhauer, B., Middeldorp, A., Zankl, H., Oostrom, V.v.: Layer systems for proving confluence. ACM Transactions on Computational Logic 16, 1-32 (2015)

12. Felgenhauer, B.: Labeling multi-steps for confluence of left-linear term rewrite systems. In: Tiwari, A., Aoto, T. (eds.) Proc. 4th IWC. pp. 33-37 (2015)

13. Hirokawa, H., Klein, D.: Saigawa: A confluence tool. In: Proc. 1st IWC. p. 49 (2012), http://www.jaist.ac.jp/project/saigawa/

14. Hirokawa, N., Nagele, J., Middeldorp, A.: Cops and CoCoWeb: Infrastructure for confluence tools. In: Proc. 9th IJCAR. LNAI, vol. 10900, pp. 346-353 (2018), http://cops.uibk.ac.at/

15. Hirokawa, N., Middeldorp, A.: Decreasing diagrams and relative termination. Journal of Automated Reasoning 47(4), 481-501 (2011)

16. Huet, G.: Confluent reductions: Abstract properties and applications to term rewriting systems. Journal of the ACM 27(4), 797-821 (1980)

17. Huet, G., Lévy, J.J.: Computations in orthogonal rewriting systems, I. In: Lassez, J.L., Plotkin, G. (eds.) Computational Logic: Essays in Honor of Alan Robinson, chap. 11. The MIT Press (1991)

18. Klop, J.: Combinatory Reduction Systems. Ph.D. thesis, Rijksuniversiteit Utrecht (1980)

19. Knuth, D.E., Bendix, P.B.: Simple word problems in universal algebras. In: Leech, J. (ed.) Computational problems in abstract algebra, Proceedings of a Conference held at Oxford under the auspices of the Science Research Council Atlas Computer Laboratory, 29th August to 2nd September 1967. pp. 263-297 (1970)

20. Liu, J.L.: Propriétés de Confluence des Règles de Réécriture par des Diagrammes Décroissants. Ph.D. thesis, Tsinghua University and l'Université Paris-Saclay préparée à l'École Polytechnique (2016) 
21. Mayr, R., Nipkow, T.: Higher-order rewrite systems and their confluence. Theoretical Computer Science 192(1), 3-29 (1998), doi :10.1016/S0304-3975(97)00143-6

22. Meseguer, J.: Conditional rewriting logic as a unified model of concurrency. Theoretical Computer Science 96, 73-155 (1992)

23. Métivier, Y.: About the rewriting systems produced by the Knuth-Bendix completion algorithm. Information Processing Letters 16(1), 31-34 (1983)

24. Nagele, J., Middeldorp, A.: Certification of classical confluence results for left-linear term rewrite systems. In: Proc. 7th ITP. LNCS, vol. 9807, pp. 290-306 (2016)

25. Ohlebusch, E.: Advanced Topics in Term Rewriting. Springer (2002)

26. Okui, S.: Simultaneous critical pairs and Church-Rosser property. In: Proc. 9th RTA. LNCS, vol. 1379, pp. 2-16 (1998)

27. Oostrom, V.v.: Confluence for Abstract and Higher-Order Rewriting. Ph.D. thesis, Vrije Universiteit, Amsterdam (Mar 1994)

28. Oostrom, V.v.: Developing developments. Theoretical Computer Science 175(1), 159-181 (1997)

29. Oostrom, V.v.: Confluence by decreasing diagrams, converted. In: Voronkov, A. (ed.) Proc. 19th RTA. LNCS, vol. 5117, pp. 306-320. Springer Berlin Heidelberg, Berlin, Heidelberg (2008), http://dx.doi.org/10.1007/978-3-540-70590-1_21

30. Rosen, B.: Tree-manipulating systems and Church-Rosser theorems. Journal of the ACM pp. 160-187 (1973)

31. Terese: Term Rewriting Systems. Cambridge University Press (2003)

32. Toyama, Y.: On the Church-Rosser property for the direct sum of term rewriting systems. Journal of the ACM 34(1), 128-143 (1987)

33. Winkler, F., Buchberger, B.: A criterion for eliminating unnecessary reductions in the Knuth-Bendix algorithm. In: Proceedings of the Colloquium on Algebra, Combinatorics and Logic in Computer Science, Volume II. Colloquia Mathematica Societatis J. Bolyai, vol. 42, pp. 849-869 (1986)

34. Yamada, A., Kusakari, K., Sakabe, T.: Nagoya Termination Tool. In: Proc. Joint 25th RTA \& 12th TLCA. pp. 466-475. LNCS (2014), https://www.trs.css.i. nagoya-u.ac.jp/NaTT/

35. Zankl, H., Felgenhauer, B., Middeldorp, A.: Labelings for decreasing diagrams. Journal of Automated Reasoning 54(2), 101-133 (2015)

\section{A Proofs omitted from or only sketched in the main text}

That having two labels suffices for Theorem 1, is an immediate consequence (give edges on the tree label 0 , others label 1 ) of the following lemma, which does not refer to decreasing diagrams.

Lemma 7. Every countable confluent rewrite relation has a spanning forest 15

Proof. Let $\rightarrow$ be countable and confluent. It suffices to show that if $\rightarrow$ has a single connected component, i.e. if $\leftrightarrow^{*}$ relates all objects, then we can construct a tree $\rightarrow \subseteq \rightarrow$ that spans $\rightarrow$ in the sense that $\rightarrow$-convertible objects have a common reduct in the tree: $\leftrightarrow^{*}=\rightarrow^{*} \cdot{ }^{*} \triangleleft-$ (and by determinism then have a least common reduct). Because of the countable confluence assumption, there is a cofinal reduction [18, i.e. a reduction $a_{0} \rightarrow a_{1} \rightarrow \ldots$ such that for all $a$, there

$\overline{15}$ Trees are connected acyclic deterministic relations, possibly infinite/without root. 
exists $i$ with $a \rightarrow a_{i}$. By removing from it any repetitions, we may assume the reduction does not contain cycles [27, Proposition 2.2.9]. Taking as initial tree $T_{0}$ this reduction, its trunk, we construct for each $j$, the tree $T_{j+1}$ by adjoining to $T_{j}$ any reduction from the $j$ th (in the countable enumeration) object $a^{j}$ to $T_{j}$, stopping at the moment we reach $T_{j}$ (possibly immediately). That a reduction from each $a^{j}$ to $T_{j}$ exists holds by cofinality of the trunk $T_{0}$ (and monotonicity of the process: $T_{j} \subseteq T_{j+1}$ for all $j$ ). To preserve being a tree, we again remove any repetitions from the adjoined reduction. That this construction is correct, yields a tree, follows from that the trunk $T_{0}$ is a straight line by construction, and that at no stage do we lose determinism: we only adjoin (edges from) nodes not yet in the tree and do not introduce cycles per construction.

Remark 4. The construction is very close to those in the literature. In particular, the trunk-construction follows [18 27], and the tree-construction follows [10]. Compared to 2710 the proof does not use a minimal distance argument, only cycle-removal. Each of the constructions first constructs the trunk and then the branches of the tree. A question is whether a spanning tree can be constructed in a single pass.

Proof (of Proposition 1). $C\left[s^{\sigma}\right]=C\left[X\left(\boldsymbol{x}^{\sigma}\right)\right]^{\llbracket X:=\lambda \boldsymbol{x} . s \rrbracket}$ with $\boldsymbol{x}$ the vector of variables in $t$.

Proposition 5. For multipatterns $\varsigma_{i}$ and $\zeta_{i}$ such that $\llbracket \varsigma_{i} \rrbracket=\llbracket \zeta_{i} \rrbracket$ for all $i$, $\varsigma_{0}^{[\boldsymbol{x}:=\boldsymbol{\varsigma}]} \sqsubseteq \zeta_{0}^{[\boldsymbol{x}:=\boldsymbol{\zeta}]}$ iff $\varsigma_{i} \sqsubseteq \zeta_{i}$ for all $i$.

Proof. Let $\varsigma=\varsigma_{0}^{[\boldsymbol{x}:=\boldsymbol{\varsigma}]}$ and $\zeta=\zeta_{0}^{[\boldsymbol{x}:=\boldsymbol{\zeta}]}$. If the 2nd-order substitutions $\sigma_{i}$ witness $\varsigma_{i} \sqsubseteq \zeta_{i}$ for all $i$, then $\bigcup_{i} \sigma_{i}$ witnesses $\varsigma \sqsubseteq \zeta$ (assuming 2nd-order variables are renamed apart). Conversely, a witnessing substitution $\sigma$ for $\varsigma \sqsubseteq \zeta$, can be decomposed into $\sigma_{i}$ as to the 2nd-order variables in the bodies of the $\zeta_{i}$.

Proof (of Proposition 4). For $\varsigma, \zeta$ multipatterns, $\varsigma \sqcup \zeta=\top$ entails by Lemma 1 that all positions in $\varsigma \sqcup \zeta$ are related via the 'has overlap' relation for patterns in $\varsigma, \zeta$. However, if also $\varsigma \sqcap \zeta=\perp$ then all patterns would be disjoint, yielding either $\varsigma=T$ and $\zeta=\perp$ or vice versa. This is impossible for patterns, as these are non-empty.

Proof (of Proposition 3). Intuitively, $\phi, \psi$ cannot be overlapped from above by other steps in $\Phi, \Psi$ because the root-positions of their contracted redexes are the same, and not from below because of $(\phi, \psi)$ being inner. Formally, by assumption the root positions $p_{\phi}$ and $p_{\psi}$ of the contracted redexes are the same. By the peak between $\Phi$ and $\Psi$ being critical, each redex-pattern in $\Phi$ overlaps some redexpattern in $\Psi$ and vice versa, as each pattern in $\Phi^{\text {src }}, \Psi^{\text {src }}$ is connected to each other such pattern in the has-overlap-with relation in their join, as shown in Lemma 1. Since $p_{\phi}=p_{\psi}$, no pattern $\omega \in \Phi, \Psi$ could overlap $\phi, \psi$ from above, i.e. has overlap with them such that $p_{\omega} \preceq_{o} p_{\phi}$. This means (using as before that terms are trees and that patterns are convex ${ }^{16}$ that in fact for every $\omega$,

${ }^{16}$ For term graphs this fails. There, due to non-convexity of patterns/left-hand sides, one may have that part of $\omega$ overlaps $\phi$ from below but at the same time $p_{\omega} \prec{ }_{o} p_{\phi}$. 
$p_{\phi} \preceq_{o} p_{\omega}$. But overlapping $\phi, \psi$ strictly from below, i.e. such that $p_{\phi}=p_{\psi} \prec_{o} p_{\omega}$ is also impossible by the choice of $\phi, \psi$ being inner. We conclude that $\omega$ is $\phi$ or $\psi$, since $\Phi$ and $\Psi$ are multisteps and the steps in a multistep are pairwise non-overlapping, from which the claim follows.

Proof (of Lemma 2). We have \let $\boldsymbol{X}=\boldsymbol{t}$ in $Z(\boldsymbol{z})^{\sigma} \rrbracket=u=\llbracket$ let $\boldsymbol{Y}=\boldsymbol{s}$ in $Z(\boldsymbol{z})^{\tau} \rrbracket$ by definition of $\sigma, \tau$ being witnesses to $\varsigma, \zeta \sqsubseteq \xi$. If there were to exist a $\sqsubseteq-$ upperbound of let $\boldsymbol{X}=\boldsymbol{t}$ in $Z(\boldsymbol{z})^{\sigma}$ and let $\boldsymbol{Y}=\boldsymbol{s}$ in $Z(\boldsymbol{z})^{\tau}$ smaller than the top $\top_{u}$ of the refinement lattice for $u$, say with witnessing substitutions $\sigma^{\prime}, \tau^{\prime}$, this would contradict $\xi$ being the join of $\varsigma, \zeta$, as updating $\sigma$ by mapping the 2ndorder variables in $Z(\boldsymbol{z})$ according to $\sigma^{\prime}$, and correspondingly updating $\tau$ by $\tau^{\prime}$, would witness an $\sqsubseteq$-upperbound of $\varsigma, \zeta$ smaller than $\xi$.

Proof (of Lemma 3). Let the multisteps $\Phi$ and $\Psi$ be given by let $\boldsymbol{Y}=\varrho(\boldsymbol{y})$ in $N$ respectively let $\boldsymbol{Z}=\boldsymbol{\theta}(\boldsymbol{z})$ in $L$, for rules $\varrho_{i}\left(\boldsymbol{y}_{\boldsymbol{i}}\right): \ell_{i} \rightarrow r_{i}$ and $\theta_{j}\left(\boldsymbol{z}_{\boldsymbol{j}}\right): g_{j} \rightarrow d_{j}$. Defining $\varsigma=\Phi^{\text {src }}, \zeta=\Psi^{\text {src }}$, and $\xi=\varsigma \sqcup \zeta$, we have $\varsigma \sqsubseteq \xi$ and $\zeta \sqsubseteq \xi$ are witnessed (see Definition (4) by some 2nd-order substitutions $\sigma=[\boldsymbol{X}:=\boldsymbol{N}]$ and $\tau=[\boldsymbol{X}:=\boldsymbol{L}]$, with $\boldsymbol{X}$ the 2nd-order variables in the body $M$ of $\xi$. If the peak is not critical, either $\xi \neq \top$ or $t$ and hence $M$ is not linear. By the assumption that $\Phi$ and $\Psi$ have overlap, $M$ must (as the meet $\sqsubseteq$-relates to it) contain at least one 2nd-order variable $X$.

If otherwise only 1st-order variables occur in $M$, then by assumption it must be non-linear, so of shape $X(\boldsymbol{w})$ having some repeated variable. Then we decompose the peak into a linear prefix and a renaming. That is, we choose $\boldsymbol{x}$ to be linear of the same length $n$ as $\boldsymbol{w}$, and define $\Phi_{0}$ and $\Psi_{0}$ as let $\boldsymbol{Y}=\boldsymbol{\varrho}(\boldsymbol{y})$ in $X(\boldsymbol{x})^{\sigma}$ respectively let $\boldsymbol{Z}=\boldsymbol{\theta}(\boldsymbol{z})$ in $X(\boldsymbol{x})^{\tau}$ (linearisations of $\Phi$ and $\Psi$ ) and $\Phi_{i}, \Psi_{i}$ both to let $=$ in $w_{i}$ (simply renaming $x_{i}$ into $w_{i}$ ).

Otherwise, we can write $M$ as $M_{0}^{\left[x_{1}:=M_{1}\right]}$ for terms $M_{i}$ in which at least one non-1st-order variable occurs (for instance, let $M_{0}$ be obtained by replacing one of the arguments of the head-symbol of $M$ by $x_{1}$ ). Then we choose the multisteps $\Phi_{i}$ and $\Psi_{i}$ to be let $\boldsymbol{Y}=\boldsymbol{\varrho}(\boldsymbol{y})$ in $M_{i}^{\sigma}$ respectively let $\boldsymbol{Z}=\boldsymbol{\theta}(\boldsymbol{z})$ in $M_{i}^{\tau}$ (and canonising the let-bindings, deleting binders for 2 nd-order variables occurring in the other body, i.e. in $M_{1-i}$ ). Again, the decomposed peaks are smaller in size.

By simple computations one verifies that in both cases the decomposed peaks compose to the original peak. Using Propositions 5 (for compositionality of $\sqcup$ and $\sqcap$ ) and 2 we compute

$$
\left.\Phi \text { ก } \Psi=\Pi\left(\Phi_{0}^{\mathrm{src}}\right)^{\left[\boldsymbol{x}:=\Phi^{\mathrm{scc}}\right]} \sqcap\left(\Psi_{0}^{\mathrm{src}}\right)^{\left[\boldsymbol{x}:=\Psi^{\mathrm{src}}\right]} \rrbracket=\sum_{i} \llbracket \Phi_{i}^{\mathrm{src}} \sqcap \Psi_{i}^{\mathrm{src}}\right\rceil=\sum_{i} \Phi_{i} \text { ก } \Psi_{i}
$$

and similarly $\Phi \uplus \Psi \geqslant \Phi_{i} \uplus \Psi_{i}$ for all $i$. In the 1st-order-variable-only case we conclude strict inequality by $\Phi \Psi \Psi=\|X(\boldsymbol{w})\|=\left(1, n^{\prime}\right)>\|X(\boldsymbol{x})\|=(1, n),\left\|w_{i}\right\|=$ $(0,1)$, for some $n^{\prime}>n$. In the other case, strict inequality follows by the choice of splitting into $M_{0}$ in $M_{1}$ in such a way that both contain at least one non-1storder variable symbol.

Proof (of Lemma 4). The 1st item holds per construction of residuals as given above. For the 2nd item, we obtain by Lemma 1, that multipatterns are the join 
of their patterns so that we can write $\Phi=\bigsqcup_{\phi \in \Phi} \phi$ and $\Psi=\bigsqcup_{\psi \in \Psi} \psi$, hence by distributivity $\Phi^{\text {src }} \sqcap \Psi^{\text {src }}=\bigsqcup_{\phi \in \Phi, \psi \in \Psi} \phi^{\text {src }} \sqcap \psi^{\text {src }}$ so that $\Phi$ and $\Psi$ have overlap iff some of their constituting steps have overlap. We conclude per construction of residuals.

Proof (of Lemma 5). Defining $\longmapsto=\rightarrow_{\mathcal{B}} \cdot \rightarrow_{\mathcal{A}}$, it suffices (cf. e.g. 31, Proposition 1.1.11]) to show $\longmapsto$ has the diamond property, as $\rightarrow \mathcal{A} \subseteq \longmapsto \subseteq \rightarrow \mathcal{A}$ by the assumption that $\rightarrow_{\mathbb{B}} \subseteq \rightarrow_{\mathcal{A}}$. We claim

$$
a \leftarrow \cdot \rightarrow_{\mathbb{B}}^{m} \cdot{ }_{\mathscr{B}}^{n} \leftarrow \cdot \rightarrow_{b} \subseteq \hookrightarrow \cdot \leftarrow
$$

holds for all labels $a, b$ and numbers $m, n \geqslant 0$. From the claim and confluence of $\rightarrow_{\mathcal{B}}$ we conclude since $\longleftarrow \cdot \longmapsto \subseteq a \leftarrow \cdot \rightarrow_{\mathcal{B}} \cdot \mathscr{B}^{\sharp} \cdot \rightarrow_{b} \subseteq \longmapsto \cdot \longleftarrow$. The claim is shown by well-founded induction on $(\{a, b\}, m+n)$ with respect to the lexicographic product of $\succ_{\text {mul }}$ and the greater-than order $>$ on $\mathbb{N}$. We distinguish cases, depending on whether or not $m+n>0$. If $m=n=0$ then

$$
a \leftarrow \cdot \rightarrow_{\mathcal{B}}^{m} \cdot n_{\mathcal{B}}^{n} \leftarrow \cdot \rightarrow_{b} \subseteq\left(\rightarrow_{\mathcal{A}} \cdot \mathcal{A} \leftarrow\right) \cup \bigcup_{\{a, b\} \succ_{\text {mul }}\left\{a^{\prime}, b^{\prime}\right\}}\left(a^{\prime} \leftarrow \cdot \leftrightarrow_{\mathcal{B}}^{*} \cdot \rightarrow_{b^{\prime}}\right) \subseteq \longmapsto \cdot \leftarrow
$$

by condition 1, and by confluence of $\rightarrow_{\mathcal{B}}$ and the I.H., respectively. Otherwise, assume w.l.o.g. that $m>0$ and consider $x_{a} \leftarrow \cdot \rightarrow_{\mathcal{B}} y \rightarrow_{\mathbb{B}}^{m-1} \cdot \cdot_{\mathscr{B}}^{n} \leftarrow \cdot \rightarrow_{b} z$. By condition 2 applied to $a \leftarrow \cdot \rightarrow_{\mathscr{B}}$ and confluence of $\rightarrow_{\mathscr{B}}$, either

$$
x \rightarrow_{\mathcal{B}} \cdot{ }_{a} \leftarrow y \rightarrow_{\mathcal{B}}^{m-1} \cdot \cdot_{\mathcal{B}}^{n} \leftarrow \cdot \rightarrow_{b} z \quad \text { or } \quad x \rightarrow_{\mathscr{B}} \cdot a^{\prime} \leftarrow \cdot \rightarrow_{\mathcal{B}} \cdot \mathscr{B}^{\leftarrow} \leftarrow \cdot \rightarrow_{b} z
$$

for some $a \succ a^{\prime}$. In both cases the I.H. applies, because of a decrement in the second component respectively a decrease in the first, and we conclude to $x \rightarrow_{\mathcal{B}} \cdot \longmapsto \cdot \hookleftarrow z$, hence to $x \longmapsto \cdot \longleftarrow z$.

Proof (of Lemma 6).

1. Specialising Definition 1 to the hot-labelling, hot-decreasingness of $s \quad \ell \leftarrow$

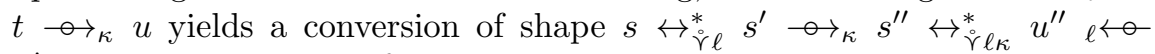
$u^{\prime} \leftrightarrow_{\curlyvee \kappa}^{*} u$, and similarly for the peak vector, where we have used that multisteps may be empty so that $\rightarrow=\rightarrow \rightarrow^{=}$, and that due to the properties of

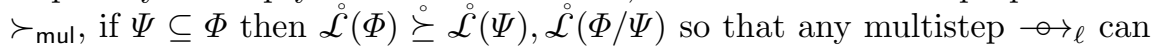
be developed into a reduction $\rightarrow_{\text {ir } \ell}$ of ordinary steps ${ }^{17}$

That all variables (which are 1st-order) occurring in the diagram may be assumed to be contained in the variables occurring in $t$, say $\boldsymbol{z}$, follows by simply substituting the same constant ${ }^{18}{ }^{19}$ for all variables not among $\boldsymbol{z}$ in the diagram. Since steps, conversions, and multisteps are closed under substitution, this preserves the shape of the diagram, and it even does not

$\overline{17}$ We may even assume [12] the multisteps are homogeneous (all rules the same label).

18 If there is no constant in the signature, as fresh constant may be adjoined without affecting confluence, as confluence is a modular property of TRSs.

${ }^{19}$ Of course, if we already know that all peaks can be completed into a decreasing diagram, then its is obvious, because then the system is confluent. 
change the peak at all: as $s, u$ are obtained from $t$ by rewriting, and rewrite rules are assumed not to introduce variables, their variables are among those of $t$. To see that the diagram is still hot-decreasing, note that if a term $p$ on the closing conversion is the source of step having a label required to be $\succ$-ordered below a source label (i.e. term) of one of the steps $\Phi, \Psi$, i.e. below $t$, then $t \rightarrow p$ from which we conclude that the variables contained in $p$ are a subset of $\boldsymbol{z}$, and if it was required to be below a rule label (i.e. set) of one of these steps, then we conclude since those labels and their order $\succ_{\text {mul }}$ are invariant under substitution of constants.

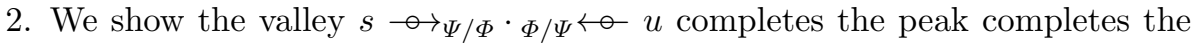
peak $s \Phi \leftarrow t \leftrightarrow \rightarrow \Psi$ into a hot-decreasing diagram. by considering all possible distributions of $C$ - and $\mathscr{R}-C$-rules in $\Phi, \Psi$. By Lemma 4 1), the rule symbols occurring in $\Psi / \Phi$ are contained in $\Psi$, and those in $\Phi / \Psi$ are contained in $\Phi$. We have on the one hand that if $\Phi$ contains some rule in $\mathcal{R}-\mathcal{C}$ then $\stackrel{\mathscr{L}}{ }(\Phi) \succeq \stackrel{\circ}{\complement}(\Phi / \Psi)$ since either $\Phi / \Psi$ contains such a rule as well so their sets of maxima are $\succeq_{\text {mul }}$-related, or else we conclude by $\succ^{\circ}$ ordering sets above terms. On the other hand, if $\Phi$ only contains $C$-rules then so does $\Phi / \Psi$ and either $\Psi$ contains some rule in $\mathscr{R}-C$ and then $\stackrel{\complement}{\mathcal{L}}(\Phi) \stackrel{\circ}{\succ}(\Phi / \Psi)$, or it does not and then $\dot{\mathcal{L}}(\Phi) \succeq \dot{\complement}(\Phi / \Psi)$ as their sources are $\rightarrow$ e-related, as desired.

3. We distinguish cases on the types of the rules in the composite peak

$$
s^{[\boldsymbol{x}:=\boldsymbol{s}]} \ell^{\prime \leftarrow \ominus-t^{[\boldsymbol{x}:=\boldsymbol{t}]}} \multimap \rightarrow_{\kappa^{\prime}} u^{[\boldsymbol{x}:=\boldsymbol{u}]}
$$

having labels as indicated.

- If either of the multisteps is empty, we conclude trivially;

- If both multisteps only contain $C$-rules, then first note that the $C$ conversions may be further restricted to be of shape $s \leftrightarrow_{\curlyvee t}^{*} u{ }^{20}$ by using that a step $t \rightarrow \ldots$ cannot occur in it. This is seen by considering what would be to the left of such a step in the conversion: it cannot be the first step since $t \neq s$ by termination of $C$; it cannot be preceded by a step $\ldots \leftarrow t$ as that would have label $t$, not a smaller one as required by decreasingness; and not by a step $\ldots \rightarrow t$ as its source cannot be smaller than $t$ as then $\rightarrow_{e}$ would be cyclic. Based on this, we construct the closing conversion 21

$$
s^{[\boldsymbol{x}:=\boldsymbol{s}]} \leftrightarrow_{i \curlyvee s^{[\boldsymbol{x}:=\boldsymbol{t}]}}^{*} s^{[\boldsymbol{x}:=\boldsymbol{u}]} \leftrightarrow_{\curlyvee}^{*}{ }_{\curlyvee}^{[\boldsymbol{x}:=\boldsymbol{u}]} u^{[\boldsymbol{x}:=\boldsymbol{u}]}
$$

It is decreasing as $t^{[\boldsymbol{x}:=\boldsymbol{t}]} \succ^{\circ} s^{[\boldsymbol{x}:=\boldsymbol{t}]}$ by $t \succ^{\circ} s$ and closure of non-empty $\mathscr{R}$ reductions under substitution, and $t^{[\boldsymbol{x}:=\boldsymbol{t}]} \succeq t^{[\boldsymbol{x}:=\boldsymbol{u}]}$ by $\boldsymbol{t} \check{\succ} \boldsymbol{u}$ and closure of $\mathscr{R}$-reductions under contexts.

- If both contain some $(\mathscr{R}-C)$-rule, then we conclude by the conversion

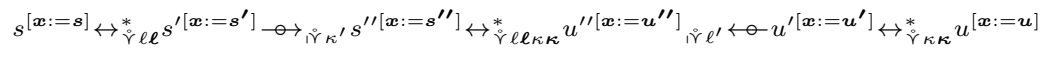

${ }_{20}$ The conversion is below $t$ (with respect to $\rightarrow_{c}^{+}$) in the sense of 33|29.

${ }^{21}$ The notation, substituting conversions at parallel positions, leaves unspecified the (sequential) order of the steps of the conversions substituted. Any choice will do. 
obtained by piecewise composing the constituents conversions, which is decreasing because $\ell \cup \ell \supseteq \ell^{\prime} \succeq_{\text {mul }} \ell, \ell$ and $\kappa \cup \kappa \supseteq \kappa^{\prime} \succeq_{\text {mul }} \kappa, \kappa$.

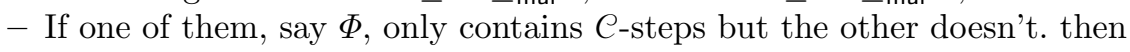
the constituent conversions have shape $s \leftrightarrow_{\curlyvee}^{*} s^{\prime} \rightarrow_{\kappa} u^{\prime} \leftrightarrow_{\curlyvee \kappa}^{*} u$ yielding

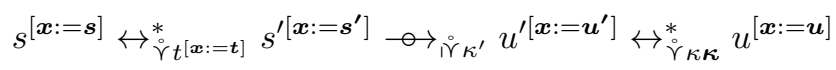

which is seen to be decreasing by reasoning as in the previous items.

Proof (of measure decrease in the induction step of Theorem 3). We have $E^{\prime}=$ $\Psi^{\prime \text { src }}$ hence $E=E^{\prime} \sqcup \psi^{\text {src }}$ as sets of patterns despite $t \neq u^{\prime}$; the positions are in both since $\psi$ is inner so the patterns $\boldsymbol{g}^{\prime}$ are not below $Y$ in $N{ }^{22}$ Using distributivity all the time, the strict inequality holds in the line of reasoning (1) by $\phi^{\text {src }} \cap \psi^{\text {src }} \neq \emptyset=\psi^{\text {src }} \cap F$, the first equality by the reasoning above and $(\phi, \psi)$ being inner so that $D_{-} \sqcap \psi^{\text {src }}=\perp$, the second since, by reasoning as for $E$, we have $D^{\prime}=\Phi^{\text {src }} \sqcup F^{\prime}$ since the patterns $\ell^{\prime}$ are not below $Z$ in $L$ by $\phi$ being inner, hence $D_{-}=\Phi^{\prime \text { src }} \sqcup F=D_{+}^{\prime}$, and the final inequality by $F \sqcap \Psi^{\prime \text { src }} \sqsupseteq F^{\prime} \sqcap \Psi^{\text {ssrc }}$ which holds because by $\hat{\Psi}$ being a multistep from $\hat{u}, F^{\prime}$ contains positions that are either below the root of $\psi$ but then not in $\Psi^{\prime}$, or in the pattern of $\phi$ and then in $F$.

We now give the idea how, as an alternative to the set-theoretic reasoning. one can also directly work on let-expressions to show that the induction measure decreases, i.e. one can proceed by giving appropriate constructions on multipatterns, and then showing that the measure decreases, constructing witnesses by computation.

For instance, one may define $D_{-}$as in the main text, but now by a letexpression, as let $\boldsymbol{X}^{\prime} Z^{\prime}=\boldsymbol{\ell}^{\prime} C\left[z^{\prime}\right]$ in $L^{\left[Z:=Z^{\prime}\left(\ell_{\phi}\right)\right]}$ for $Z^{\prime}, z^{\prime}$ fresh. Here $C$ is $E$ as defined above, but now constructed from the image $C\left[Y\left(\ell_{\phi}\right)\right]$ of $Z$ under the 2ndorder substitution $\tau$ witnessing $\psi^{\text {src }} \sqsubseteq \varsigma$, where $\varsigma$ in turn was constructed as the join of $\psi$ and $\Phi$. For another example, $F$, the part of the pattern of $\phi$ that does not belong to the pattern of $\phi$, can be constructed by let $Z^{\prime}=C\left[z^{\prime}\right]$ in $L^{\left[\boldsymbol{X}^{\prime} Z:=\ell^{\prime} Z^{\prime}\left(\boldsymbol{\ell}_{\phi}\right)\right]}$.

The same reasoning applies, but now by computation on multipatterns. For instance, using distributivity to decompose $\Phi, \Psi$ in their constituent steps, the inequality on the amount of overlap follows from $\llbracket \phi^{\mathrm{scc}} \sqcap \psi^{\mathrm{src}} \sqcap \pi>\pi \perp \rrbracket=\pi \phi^{\mathrm{scc}} \sqcap$ $\perp \rrbracket=\llbracket \phi^{\mathrm{scc}} \sqcap \psi^{\mathrm{src}} \sqcap D_{-} \rrbracket$ with $\psi^{\mathrm{src}} \sqcap D_{-}=\perp$ by their complementary definition via $C$. Similarly, one may proceed from the right.

A difference in the reasoning shows up 'in the middle' of the line of reasoning (1): since let-expression can only be compared with respect to the refinement order $\sqsubseteq$ if they denote the same term, peaks for different terms, as is the case here, can a priori not be compared. The way around this is to use Proposition 2 to split-off any differing (non-patterns) parts first. For instance, the amounts of overlap (let $X=f(f(x))$ in $X(f(a)))$ ก (let $Y=f(f(x))$ in $f(Y(a))$ ) and (let $X=f(f(x))$ in $X(f(b)))$ ก (let $Y=f(f(x))$ in $f(Y(b))$ ) are clearly the same; the $a$ and $b$ are innocuous here. That can be implemented for let-expressions

${ }^{22}$ In the let-expression representation this follows from Proposition 2 by vertically decomposing both having as substitute the redex respectively the contractum of $\psi$. 
by vertically decomposing the let-expression involved. For instance, decomposing the first as (let $X=f(f(x))$ in $X(f(x)))^{[x:=(\text { let }=\text { in } a)]}$. Since the total amount of overlap is the sum of that of the components, the substitutes have no overlap, and the prefixes now have the same denotations, we may proceed, and have recovered the possibilities of the set-theoretic representation on let-expressions. 